\title{
Muon spin relaxation and neutron scattering investigations of the noncentrosymmetric heavy-fermion antiferromagnet $\mathrm{CeRhGe}_{3}$
}

\author{
A. D. Hillier, ${ }^{1,}{ }^{*}$ D. T. Adroja, ${ }^{1, \dagger}$ P. Manuel, ${ }^{1}$ V. K. Anand, ${ }^{1}$ J. W. Taylor, ${ }^{1}$ K. A. McEwen, ${ }^{2}$ B. D. Rainford, ${ }^{3}$ and M. M. Koza ${ }^{4}$ \\ ${ }^{1}$ ISIS Facility, STFC, Rutherford Appleton Laboratory, Chilton, Oxfordshire, OX11 0QX, United Kingdom \\ ${ }^{2}$ Department of Physics and Astronomy, and London Centre for Nanotechnology, University College London, Gower Street, \\ London WCIE 6BT, United Kingdom \\ ${ }^{3}$ Department of Physics and Astronomy, Southampton University, Southampton, SO17 1BJ, United Kingdom \\ ${ }^{4}$ Institut Laue Langevin, BP 156, 38042 Grenoble Cedex 9, France
}

(Received 24 August 2011; revised manuscript received 16 February 2012; published 2 April 2012)

\begin{abstract}
The magnetic ground state of $\mathrm{CeRhGe}_{3}$ has been investigated using magnetic susceptibility, heat capacity, neutron diffraction, muon spin relaxation $(\mu \mathrm{SR})$, and inelastic neutron scattering (INS) techniques. Our $\mu \mathrm{SR}$ study clearly reveals the presence of two frequencies below $\mathrm{T}_{\mathrm{N} 2}=7 \mathrm{~K}$ and three frequencies between $7 \mathrm{~K}$ and $\mathrm{T}_{\mathrm{N} 1}=14.5 \mathrm{~K}$, indicating long-range magnetic ordering of the $\mathrm{Ce}^{3+}$ moment. The temperature dependence of the highest frequency follows a mean-field order parameter. Our powder neutron diffraction study at $1.5 \mathrm{~K}$ reveals the presence of magnetic Bragg peaks, indexed by the propagation vector $\mathrm{k}=(0,0,3 / 4)$ with the $\mathrm{Ce}^{3+}$ magnetic moment $\sim 0.45(9) \mu_{\mathrm{B}}$ along the $c$ axis. INS studies at $18 \mathrm{~K}$ (i.e., above $\mathrm{T}_{\mathrm{N} 1}$ ) show the presence of two well-defined crystal-field (CEF) excitations at 7.5 and $18 \mathrm{meV}$. At 10 and $4.5 \mathrm{~K}$, a very small increase has been observed in the CEF excitation energies. At $100 \mathrm{~K}$, both CEF excitations broaden and a broad quasielastic component has also been observed. Further, the low-energy INS study reveals the presence of a nearly temperature-independent quasielastic linewidth between 16 and $60 \mathrm{~K}$, which indicates a Kondo temperature $\mathrm{T}_{\mathrm{K}}=12.6(3) \mathrm{K}$. The presence of well-defined $\mathrm{CEF}$ excitations in $\mathrm{CeRhGe}_{3}$ suggests local moment magnetism and may explain the absence of pressure-induced superconductivity. Analyzing the INS data based on a CEF model, we have evaluated the CEF ground-state wave functions and ground-state moment. The observed small value of the ordered moment along the $c$ axis, deduced from the neutron diffraction data, contrasts with the ab-plane moment direction predicted by the single-ion CEF anisotropy and indicates the presence of two-ion anisotropic magnetic exchange interactions, which govern the direction of the moment.
\end{abstract}

DOI: 10.1103/PhysRevB.85.134405

PACS number(s): 75.30.Mb, 75.10.Dg, 75.20.Hr, 75.30.Gw

\section{INTRODUCTION}

Strongly correlated electron systems, displaying heavyfermion (HF) behavior, have attracted considerable attention both in theoretical and experimental condensed matter physics due to the many exotic properties they can exhibit. ${ }^{1-4}$ One of the most attractive and elusive phenomena of these HF systems is the coexistence of magnetism and superconductivity (SC), which is at the forefront of condensed matter research. The SC in HF systems cannot be explained by the BardeenCooper-Schrieffer (BCS) theory relevant for conventional $s$-wave phonon-mediated superconductors. Many HF compounds, having centrosymmetric crystal structures, exhibit unconventional SC close to a quantum critical point (QCP), where magnetic order disappears and SC emerges. The region where SC exists can be reached as a function of pressure, magnetic field, or alloying, implying that magnetic fluctuations are playing an important role in the formation of the Cooper pairs. ${ }^{3}$ In centrosymmetric materials, the spin and orbital parts of the pair wave function can be treated individually because the conduction bands are degenerate even if the spin-orbit coupling is strong. ${ }^{2}$ Therefore, one can express the Cooper pair as either a spin-singlet or a spin-triplet pairing. The pairing mechanism in heavy-fermion superconductors (HFSC) is not yet understood, as is also the case in the high $T_{c}$ cuprates (HTSC) and the newly discovered Fe-based superconductors. ${ }^{3,4}$ One of the current hypotheses for these unconventional superconductors is that the superconducting pairing is mediated by magnetic interactions, the so-called "magnetic glue."3,5 Despite large volumes of data available in the literature on HTSC and HFSC, the origin of the SC and pairing mechanism in these classes of materials remains difficult to understand.

Recently, we have been investigating the physical properties of $R \mathrm{TX}_{3}(\mathrm{R}=$ rare earth, $\mathrm{T}=$ transition metal, $\mathrm{X}=\mathrm{Si}, \mathrm{Ge}, \mathrm{Sn})$ compounds using $\mu \mathrm{SR}$ and neutron scattering techniques. ${ }^{6-9}$ Ce compounds of this series, $\mathrm{CeTX}_{3}(\mathrm{X}=\mathrm{Si}$ and $\mathrm{Ge})$, belong to the family of recently discovered noncentrosymmetric HFSC whose superconducting ground-state properties are dictated by an antisymmetric spin-orbit coupling (ASOC) as a consequence of the lack of inversion symmetry, along the $\left[\begin{array}{lll}0 & 0 & 1\end{array}\right]$ direction, in the tetragonal crystal structure. ${ }^{10-19}$ This ASOC, in turn, results in the formation of a mixed pairing wave function with spin-singlet and spin-triplet components in the superconducting state. The relationship between SC and the lack of inversion symmetry is therefore a central issue, which needs to be clarified both theoretically and experimentally in f-electron systems. Among these systems, $\mathrm{CeTX}_{3}$ compounds exhibit many interesting properties (see Table I). For example, at ambient pressure $\mathrm{CeCoSi}_{3}$ shows $\mathrm{SC}$ at $0.8-1.3 \mathrm{~K},{ }^{10,11}$ while $\mathrm{CeRhSi}_{3}$ and $\mathrm{CeIrSi}_{3}$ order antiferromagnetically (AFM) at 1.5 and $5 \mathrm{~K}$, respectively, ${ }^{12-15}$ and also reveal SC over a broad range of applied pressures below 1.1 and $1.6 \mathrm{~K}$, respectively. ${ }^{12-15}$

The Ge-based compounds $\mathrm{CeTGe}_{3}$ also exhibit interesting magnetic and superconducting properties. ${ }^{16-18} \mathrm{CeRhGe}_{3}$ and $\mathrm{CeIrGe}_{3}$ exhibit three successive magnetic phase transitions, 
TABLE I. A summary of the physical properties of $\mathrm{CeTX}_{3}$ compounds crystallizing in the $\mathrm{BaNiSn}_{3}$-type noncentrosymmetric tetragonal crystal structure ( $I 4 \mathrm{~mm}$, No. 107): the magnetic ground state, superconducting transition temperature $\left(\mathrm{T}_{c}\right)$ at pressure $(\mathrm{P})$, paramagnetic Curie Weiss temperature $\left(\theta_{\mathrm{P}}\right)$, electronic specific-heat coefficient $(\gamma)$, Kondo temperature $\left(\mathrm{T}_{\mathrm{K}}\right)$, and residual resistivity $\left(\rho_{0}\right)$. The magnetic ground states are antiferromagnetic (AFM) with Néel temperature $\mathrm{T}_{\mathrm{N}}$, paramagnetic (PM), and spin glass (SG). VF and HF denote valence fluctuation and HF states, respectively.

\begin{tabular}{|c|c|c|c|c|c|c|c|c|}
\hline Compound magnetism & $\begin{array}{c}\theta_{\mathrm{P}} \\
(\mathrm{K})\end{array}$ & $\begin{array}{l}\mathrm{T}_{\mathrm{N}} \\
(\mathrm{K})\end{array}$ & $\begin{array}{c}\gamma \\
\mathrm{mJ} / \mathrm{mol} \mathrm{K}^{2}\end{array}$ & $\begin{array}{l}\mathrm{T}_{\mathrm{K}} \\
(\mathrm{K})\end{array}$ & $\begin{array}{c}\mathrm{T}_{c} \\
(\mathrm{~K})\end{array}$ & $\begin{array}{c}\mathrm{P} \\
\text { (kbar) }\end{array}$ & $\begin{array}{c}\rho_{0} \\
(\mu \Omega \mathrm{cm})\end{array}$ & Ref. \\
\hline $\mathrm{CeCoSi}_{3} \mathrm{PM}(\mathrm{VF})$ & -840 & & 37 & 900 & $0.7-1.4$ & 0 & 12 & $10,11,54$ \\
\hline $\mathrm{CeRuSi}_{3} \mathrm{PM}(\mathrm{VF})$ & & & & & & & $\sim 28$ & 10 \\
\hline $\mathrm{CeRhSi}_{3} \mathrm{AFM}(\mathrm{HF})$ & -128 & 1.6 & 120 & 100 & 1 & 23.1 & $\sim 6$ & 12,16 \\
\hline $\mathrm{CePdSi}_{3} \mathrm{AFM}$ & 26 & $3 / 5.2$ & 57 & & & & $\sim 2$ & 55 \\
\hline $\mathrm{CeOsSi}_{3} \mathrm{PM}(\mathrm{VF})$ & & & & & & & $\sim 20$ & 10 \\
\hline $\mathrm{CeIrSi}_{3} \mathrm{AFM}(\mathrm{HF})$ & -142 & 5.0 & 125 & 100 & 1.6 & 25 & $\sim 10$ & 14,16 \\
\hline $\mathrm{CePtSi}_{3} \mathrm{AFM}$ & $-211 / 12$ & $4.8 / 2.4$ & 29 & & & & 16.6 & 19 \\
\hline $\mathrm{CeFeGe}_{3} \mathrm{PM}(\mathrm{HF})$ & -90 & & 150 & 100 & & & $\sim 8$ & 56 \\
\hline $\mathrm{CeCoGe}_{3} \mathrm{AFM}$ & $-71 /-29$ & $21 / 12 / 832$ & & & & & $1 / 2.5$ & 20 \\
\hline $\mathrm{CeRhGe}_{3} \mathrm{AFM}$ & -28 & $14.6 / 10 / 0.55$ & 40 & & & & $\sim 2\left(10^{\mathrm{a}}\right)$ & 16 \\
\hline $\mathrm{CeIrGe}_{3} \mathrm{AFM}$ & -21 & $8.7 / 4.7 / 0.7$ & 80 & & & & $\sim 5$ & 16 \\
\hline${ }^{\mathrm{b}} \mathrm{CeNiGe}_{3} \mathrm{AFM}$ & -17 & 5.5 & 45 & 4.3 & 0.48 & 65 & $\sim 8$ & 22,57 \\
\hline${ }^{\mathrm{c}} \mathrm{CeRuGe}_{3} \mathrm{SG}(\mathrm{HF})$ & -7.5 & & 428 & & & & & 58 \\
\hline
\end{tabular}

${ }^{\text {a Present work. }}$

${ }^{\mathrm{b}}$ Crystallizes in the orthorhombic $\mathrm{SmNiGe}_{3}$-type structure (Cmmm, No. 65).

${ }^{\mathrm{c}}$ Crystallizes in the cubic structure (Pm3n, No. 223).

$\mathrm{T}_{\mathrm{N} 1}=14.6 \mathrm{~K}, \mathrm{~T}_{\mathrm{N} 2}=7 \mathrm{~K}, \mathrm{~T}_{\mathrm{N} 3}=0.55 \mathrm{~K}$ for $\mathrm{CeRhGe}_{3}$ and $\mathrm{T}_{\mathrm{N} 1}=8.7 \mathrm{~K}, \mathrm{~T}_{\mathrm{N} 2}=4.7 \mathrm{~K}, \mathrm{~T}_{\mathrm{N}}=0.7 \mathrm{~K}$ for $\mathrm{CeIrGe}_{3}$, respectively. ${ }^{16,17} \mathrm{~T}_{\mathrm{N} 1}$ for $\mathrm{CeRhGe}_{3}$ increases with increasing pressure and reaches a maximum of $21.3 \mathrm{~K}$ at $8.0 \mathrm{GPa} .{ }^{17}$ Whereas for $\mathrm{CeIrGe}_{3}, \mathrm{~T}_{\mathrm{N} 1}$ remains nearly constant, but $\mathrm{T}_{\mathrm{N} 2}$ increases with pressure and merges with $\mathrm{T}_{\mathrm{N} 1}$ at $4 \mathrm{GPa} .{ }^{17} \mathrm{Upon}$ increasing the pressure still further, $\mathrm{SC}$ has been observed in $\mathrm{CeIrGe}_{3}$ above $20 \mathrm{GPa}$ with a transition temperature of $1.6 \mathrm{~K}$ at $24 \mathrm{GPa}^{18}$ Interestingly, $\mathrm{CeCoGe}_{3}$ also exhibits unusual magnetic properties with an ordering temperature as high as $21 \mathrm{~K}^{20}$ and pressure-induced $\mathrm{SC}\left(\mathrm{T}_{\mathrm{sc}}=0.7 \mathrm{~K}\right.$ at $\left.5.5 \mathrm{GPa}\right){ }^{21}$ Furthermore, the Kondo lattice system $\mathrm{CeNiGe}_{3}$, which crystallizes in the orthorhombic space group $(\mathrm{Cmmm})$, orders antiferromagnetically below $5.5 \mathrm{~K}$ and exhibits pressureinduced HFSC, showing two domes of SC with a maximum $\mathrm{T}_{\mathrm{sc}}=0.3 \mathrm{~K}$ around $3.5 \mathrm{GPa}$ and $0.4 \mathrm{~K}$ around $7 \mathrm{GPa}$ and Fermi liquid behavior at higher pressure..$^{22,23}$

To gain insight into the low-temperature physical properties, anisotropy, and strength of hybridization in $\mathrm{CeRhGe}_{3}$, the magnetic and thermal properties have been investigated using various experimental techniques. The aim of the current study is to provide a direct comparison of this compound with $\mathrm{CeRhSi}_{3}, \mathrm{CeIrSi}_{3}$, and $\mathrm{CeIrGe}$, which exhibit noncentrosymmetric HFSC under the application of pressure. To develop any realistic theoretical model of magnetism and SC, information on the magnetic structure and CEF groundstate wave functions is important. Our $\mu$ SR and neutron diffraction studies clearly reveal long-range magnetic ordering in $\mathrm{CeRhGe}_{3}$ below $14.5 \mathrm{~K}$. Inelastic neutron scattering (INS) reveals two well-defined crystal-field (CEF) excitations at 7.5 and $18 \mathrm{meV}$, indicating the localized nature of the $4 \mathrm{f}$-electrons in $\mathrm{CeRhGe}_{3}$.

\section{EXPERIMENTAL DETAILS}

Polycrystalline samples (mass $\sim 7 \mathrm{~g}$ ) of $\mathrm{CeRhGe}_{3}$ and its nonmagnetic phonon reference $\mathrm{LaRhGe}_{3}$ were prepared by the standard arc-melting method starting with a stoichiometric mixture of the high-purity elements (Ce, La: 99.9\%, Rh: $99.99 \%$, Ge: $99.999 \%$ ) on a water-cooled copper hearth under an inert argon atmosphere. To improve the homogeneity and phase purity, the samples were flipped and remelted several times during the preparation process. The as-cast samples were wrapped in tantalum foils and annealed for 1 week at $950{ }^{\circ} \mathrm{C}$ under a dynamic vacuum to improve the phase formation. Magnetic susceptibility was measured using a commercial superconducting quantum interference device (SQUID) magnetometer (MPMS, Quantum Design). Specific heat measurements were performed by the relaxation method in a physical properties measurement system (PPMS, Quantum Design).

The neutron scattering and muon spin relaxation $(\mu \mathrm{SR})$ experiments were carried out at the ISIS Pulsed Neutron and Muon Facility of the Rutherford Appleton Laboratory, United Kingdom. The neutron diffraction measurements were carried out on the general materials (GEM) (at $300 \mathrm{~K}$ to check the phase purity) and $\mathrm{WISH}^{24}$ (at 1.5 and $20 \mathrm{~K}$ to determine the magnetic structure) time-of-flight diffractometers. The powdered sample was put into a vanadium can with a diameter of $6 \mathrm{~mm}$ and placed inside a standard helium cryostat on WISH, and the measuring time was $6 \mathrm{~h}$ at each temperature. The INS measurements were carried out on the time-of-flight (TOF) MARI spectrometer between 4.5 and $100 \mathrm{~K}$. The powder samples were wrapped in a thin $\mathrm{Al}$ foil and mounted inside a thin-walled cylindrical $\mathrm{Al}$ can, which was cooled down to $4.5 \mathrm{~K}$ inside a top-loading closed-cycle refrigerator with He-exchange gas around the samples. The measurements were 
performed with an incident neutron energy $\mathrm{E}_{i}=45 \mathrm{meV}$, corresponding to a full width at half maximum (FWHM) instrumental resolution of 1.66 and $1.0 \mathrm{meV}$ at zero and $20 \mathrm{meV}$ energy transfer, respectively. Additional low-energy neutron scattering measurements were carried out using the TOF spectrometer IN6 at the Institut Laue-Langevin (ILL), Grenoble, France with $\mathrm{E}_{i}=3.1 \mathrm{meV}$ (elastic energy resolution $0.07 \mathrm{meV}$ ) and $4.86 \mathrm{meV}$ (elastic energy resolution $0.17 \mathrm{meV}$ ). The $\mu \mathrm{SR}$ experiments were performed on the MuSR spectrometer in the longitudinal geometry configuration. At ISIS, pulses of muons are implanted into the sample at $50 \mathrm{~Hz}$ and with a FWHM of $\sim 70$ ns. These implanted muons decay with a half-life of $2.2 \mu$ s into positrons, which are emitted preferentially in the direction of the muon spin axis. Each positron is time stamped, and therefore the muon polarization can be followed as a function of time. The MuSR spectrometer comprises 64 detectors. The detectors before the sample (F) are summed together as well as the detectors after the sample (B). The muon polarization then can be determined by

$$
G_{z}(t)=\frac{N_{F}(t)-\alpha N_{B}(t)}{N_{F}(t)+\alpha N_{B}(t)},
$$

where $\mathrm{N}_{\mathrm{F}}$ and $\mathrm{N}_{\mathrm{B}}$ are the counts in the forward and backward detectors, respectively, and $\alpha$ is a calibration constant. The sample was mounted on an Ag plate and covered with a thin layer of Mylar. The sample mount was then inserted into an Oxford Instruments Variox cryostat with a temperature range of 1.2 to $300 \mathrm{~K}$. Any Ag exposed to the muon beam would give a time-independent background.

\section{RESULTS AND DISCUSSION}

\section{A. Neutron diffraction: Characterization at room temperature}

Neutron powder diffraction patterns of both samples at $300 \mathrm{~K}$ were collected on the GEM diffractometer, and the analysis was carried out using the GSAS program on the basis of a tetragonal $\mathrm{BaNiSn}_{3}$-type structure (space group I4 $m m$, No. 107). In this structure, the $\mathrm{Ce}$ (or La) and $\mathrm{Rh}$ atoms occupy the $2 \mathrm{a}$ sites $\left(\begin{array}{lll}0 & 0 \mathrm{z}\end{array}\right)$, where $\mathrm{z}=\mathrm{z}_{\mathrm{Ce}}\left(\right.$ or $\left.\mathrm{z}_{\mathrm{La}}\right), \mathrm{z}_{\mathrm{Rh}}, \mathrm{Ge} 1$ atoms occupy the $2 \mathrm{a}\left(\begin{array}{lll}0 & 0 & 0\end{array}\right)$, while the Ge 2 atoms occupy the $4 \mathrm{~b}\left(0 \frac{1}{2} \mathrm{z}_{\mathrm{Ge} 2}\right)$ sites.

A summary of our results is given in Table II, and these values are in agreement with the published data. ${ }^{17,25}$ Our analysis showed that both the samples were nearly single phase. Additionally, when the site occupancy of the Ce and Ge atoms was varied, while keeping the site occupancy of the Rh atom fixed to $100 \%$, we found no improvement in the weighted profile reliability factor $\left(\mathrm{R}_{\mathrm{WP}}\right)$, but the site occupancies of $\mathrm{Ce}$ (La) and Ge were found to be about 92 and $98 \%$. As the $\mathrm{R}_{\mathrm{WP}}$ did not improve, we kept all occupancies fixed to $100 \%$ in the final refinement. The nearest-neighbor distances are (in $\AA$ ) 4.3960 for Ce-Ce, 3.4226 for Ce-Rh, 3.2104 for Ce-Ge1, 3.2498 for $\mathrm{Ce}-\mathrm{Ge} 2$, and 2.3900 for Rh-Ge1. The distance between $\mathrm{Rh}-\mathrm{Ge} 1$ is the shortest among these distances. Further, the four $\mathrm{Ge} 1$ atoms are at the shortest distance from the $\mathrm{Ce}$, which suggests the importance of $\mathrm{Ce}-4 \mathrm{f}$ and $\mathrm{Ge}-4 \mathrm{p}$ hybridization on the physical properties of $\mathrm{CeRhGe}_{3}$ and could explain why the superconducting properties of the $\mathrm{Si}$-based compounds, $\mathrm{CeTSi}_{3}(\mathrm{~T}=\mathrm{Co}, \mathrm{Rh}$ and $\mathrm{Ir})$, are different from those of the Ge-based compounds.

\section{B. Magnetic susceptibility}

The dc susceptibility of $\mathrm{CeRhGe}_{3}$ was measured between 2 and $300 \mathrm{~K}$ in an applied magnetic field of $0.1 \mathrm{~T}$ with the results shown in Fig. 1(a). The low-temperature susceptibility [see the inset in Fig. 1(a)] shows an anomaly near $14.5 \mathrm{~K}$ and a peak at $8.5 \mathrm{~K}$, indicating two magnetic phase transitions in agreement with previous reports. ${ }^{17}$ The slight upturn below $3 \mathrm{~K}$ is due to another (third) magnetic transition at $0.55 \mathrm{~K}$, which is also seen by Muro et al. (see the inset of Fig. 5 in Ref. 16). For temperatures above $150 \mathrm{~K}$, the inverse susceptibility follows a Curie-Weiss $(\mathrm{CW})$ behavior, with an effective paramagnetic moment, $\mu_{\mathrm{eff}}=2.30(1) \mu_{\mathrm{B}}$ and a $\mathrm{CW}$ temperature $\theta_{\mathrm{P}}=$ $+3.1(1) \mathrm{K}$. The small, but positive, value of $\theta_{\mathrm{P}}$ may be due to either weak ferromagnetic correlations between the $\mathrm{Ce}$ ions in the high-temperature range or the presence of CEF and anisotropic two-ion exchange. Support for the latter hypothesis comes from our analysis of the single-crystal susceptibility

TABLE II. A summary of the results obtained from the refinement of the room-temperature neutron powder diffraction data: the lattice parameters, atomic positions, occupancy, and thermal parameters. The site occupancy for all the atoms was fixed to $100 \%$. The reliability factors (weighted profile factor $\mathrm{R}_{\mathrm{wp}}$ ) were calculated by comparing the fit to the data.

\begin{tabular}{|c|c|c|c|c|}
\hline & $\mathrm{CeRhGe}_{3}$ & $\mathrm{LaRhGe}_{3}$ & & \\
\hline Space group & $I 4 \mathrm{~mm}$ & $14 \mathrm{~mm}$ & & \\
\hline $\mathrm{a}(\AA)$ & $4.39604(2)$ & $4.41978(2)$ & & \\
\hline c $(\AA)$ & $10.02243(7)$ & $10.04588(7)$ & & \\
\hline \multirow{2}{*}{$\mathrm{R}_{\mathrm{wp}}(\%)$} & 5.05 & 4.71 & & \\
\hline & $\mathrm{x}$ & $\mathrm{y}$ & $\mathrm{z}$ & $U\left(\times 10^{-2}\right) \AA^{2}$ \\
\hline$\overline{\mathrm{Ce}}$ & 0 & 0 & $0.58006(7)$ & $0.887(16)$ \\
\hline $\mathrm{Rh}$ & 0 & 0 & $0.23847(5)$ & $0.049(13)$ \\
\hline Ge1 & 0 & 0 & 0 & $0.701(11)$ \\
\hline $\mathrm{Ge} 2$ & 0 & 0.5 & $0.34121(4)$ & $0.817(9)$ \\
\hline $\mathrm{La}$ & 0 & 0 & $0.58138(7)$ & $0.681(12)$ \\
\hline $\mathrm{Rh}$ & 0 & 0 & $0.23949(9)$ & $0.012(10)$ \\
\hline Ge1 & 0 & 0 & 0 & $0.626(11)$ \\
\hline $\mathrm{Ge} 2$ & 0 & 0.5 & $0.34204(6)$ & $0.720(9)$ \\
\hline
\end{tabular}




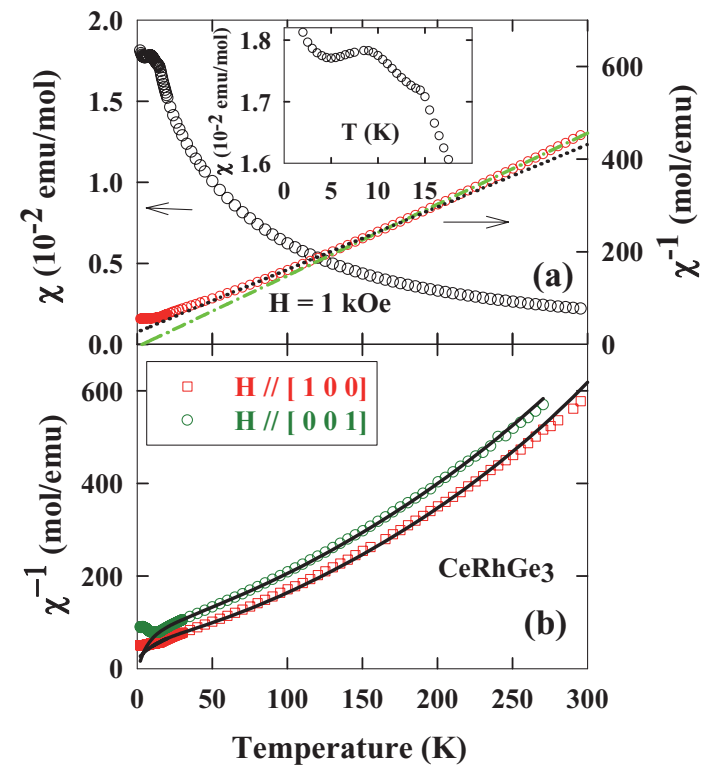

FIG. 1. (Color online) (a) Temperature dependence of the magnetic susceptibility (left $y$ axis) and the inverse magnetic susceptibility (right $y$ axis) of polycrystalline $\mathrm{CeRhGe}_{3}$. The dotted and dash-dotted lines show fits to $\mathrm{CW}$ behavior in the low- and high-temperature regions, respectively. The inset figure shows the low-temperature behavior of the susceptibility. (b) Temperature dependence of the inverse magnetic susceptibility of $\mathrm{CeRhGe}_{3}$ single crystal from Ref. 17. The solid lines show the fit based on the crystal-field model (together with INS data) including anisotropic molecular field parameters $\left(\lambda^{\xi}\right)$ and temperature-independent constant susceptibility $\left(\chi_{0}^{\xi}\right)$. The form of the susceptibility used in the direction $\xi(=\perp$ and $\|$ to $c$ axis $)$ is given by $\chi^{\xi}=\chi_{\mathrm{CEF}}^{\xi} /\left(1-\lambda^{\xi} \chi_{\mathrm{CEF}}^{\xi}\right)+\chi_{0}^{\xi}$, where $\chi_{\mathrm{CEF}}^{\xi}$ is the single-ion susceptibility calculated using the CEF model. The values of the parameters obtained from the fit are given in Table III.

discussed in Sec. IIIF. The observed value of $\mu_{\text {eff }}$ is slightly smaller than $2.54 \mu_{\mathrm{B}}$ expected for a $\mathrm{Ce}^{3+}$ ion with $\mathrm{J}=5 / 2$. Further, as can be seen from Fig. 1(a), the inverse susceptibility also exhibits CW-type behavior between 30 and $135 \mathrm{~K}$ but with $\mu_{\text {eff }}=2.56(2) \mu_{\mathrm{B}}$ and $\theta_{\mathrm{P}}=-31.8(3) \mathrm{K}$. The value of $\mu_{\text {eff }}$ estimated from the low-temperature CW behavior is similar to that expected for a $\mathrm{Ce}^{3+}$ ion. The large and negative value of $\theta_{\mathrm{P}}$ indicates AFM interactions between the Ce ions. Such a large negative value of $\theta_{\mathrm{P}}$ has also been observed in many $\mathrm{Ce}$ - and $\mathrm{Yb}$-based $\mathrm{HF}$ systems and provides an estimate of the Kondo temperature through $\mathrm{T}_{\mathrm{K}} \sim\left|\theta_{\mathrm{P}}\right| / 4.5$ based on the s-d model. ${ }^{26}$ Further within the symmetric Anderson model and local moment regime, Krishnamurthy et al. derived the relation $\mathrm{T}_{\mathrm{K}}=\left|\theta_{\mathrm{P}}\right| / 2 .{ }^{27}$ From our susceptibility data and using the s-d model, we obtain $\mathrm{T}_{\mathrm{K}}=7 \mathrm{~K}$ for $\mathrm{CeRhGe}_{3}$. It should be noted that a similar method for estimation of $\mathrm{T}_{\mathrm{K}}$ (estimated from $\left.\left|\theta_{\mathrm{P}}\right| / 4\right)$ has also been used for $\mathrm{CeCuAl}_{3}$ and $\mathrm{CeMg}_{3}{ }^{28,29}$

It is worth mentioning here that the single-crystal susceptibility of $\mathrm{CeRhGe}_{3}{ }^{17}$ exhibits an anisotropy with the easy axis along [lll $\left.\begin{array}{lll}1 & 0 & 0\end{array}\right]$ [see Fig. 1(b)]. More specifically, the inverse susceptibility of a single crystal in both $\left[\begin{array}{lll}1 & 0 & 0\end{array}\right]$ and $\left[\begin{array}{lll}0 & 0 & 1\end{array}\right]$ directions exhibits $\mathrm{CW}$ behavior in two distinct temperature ranges, between 150 and $300 \mathrm{~K}$ and between 30 and $140 \mathrm{~K}$ [Fig. 1(b)]. The estimates of the effective paramagnetic moments and $\mathrm{CW}$ temperatures are $\mu_{\text {eff }}=$
$1.9 \mu_{\mathrm{B}}\left(1.9 \mu_{\mathrm{B}}\right)$ and $\theta_{\mathrm{P}}=48.3 \mathrm{~K}(27.8 \mathrm{~K})$ along $\left[\begin{array}{lll}1 & 0 & 0\end{array}\right]$ ([ $\left.\left.\begin{array}{lll}0 & 0 & 1\end{array}\right]\right)$ for the high-temperature range and $\mu_{\mathrm{eff}}=2.4 \mu_{\mathrm{B}}$

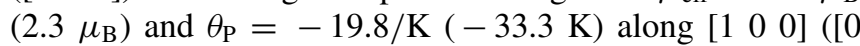
0 1]) for the low-temperature range. ${ }^{17} \mathrm{We}$, therefore, see some commonality with our powder measurements, namely, the existence of two $\mathrm{CW}$ regions with ferromagnetic-type interactions and reduced moments in the high-temperature range, while the low-temperature range displays AFM-type interactions and effective moments closer to that expected for a $\mathrm{Ce}^{3+}$ ion. The presence of two different regions over which $\mathrm{CW}$ behavior is observed may suggest either the CEF parameters are temperature dependent, as observed in $\mathrm{CeCuSi},{ }^{30}$ or the presence of anisotropic two-ion exchange interactions. Analysis of the single-crystal susceptibility [fit shown by solid lines in Fig. 1(b)] was carried out along with INS data and further discussion on this is given in Sec. III F. It is interesting to note that $\theta_{\mathrm{P}}$ deduced from the single-crystal susceptibility is higher along $\mathrm{c}$ than along a.

\section{Heat capacity}

The heat capacity of $\mathrm{CeRhGe}_{3}$ is shown in Fig. 2(a), together with that of the nonmagnetic phonon reference $\mathrm{LaRhGe}_{3}$. The latter compound does not exhibit any anomaly or onset of SC down to $2 \mathrm{~K}$. The low-temperature behavior of the heat capacity of $\mathrm{LaRhGe}_{3}$ follows the form $\mathrm{C}_{p}(\mathrm{~T}) / \mathrm{T}=$ $\gamma+\beta \mathrm{T}^{2}$, indicating a phonon contribution to the heat capacity. Fitting the data below $6.0 \mathrm{~K}$, we have estimated the value

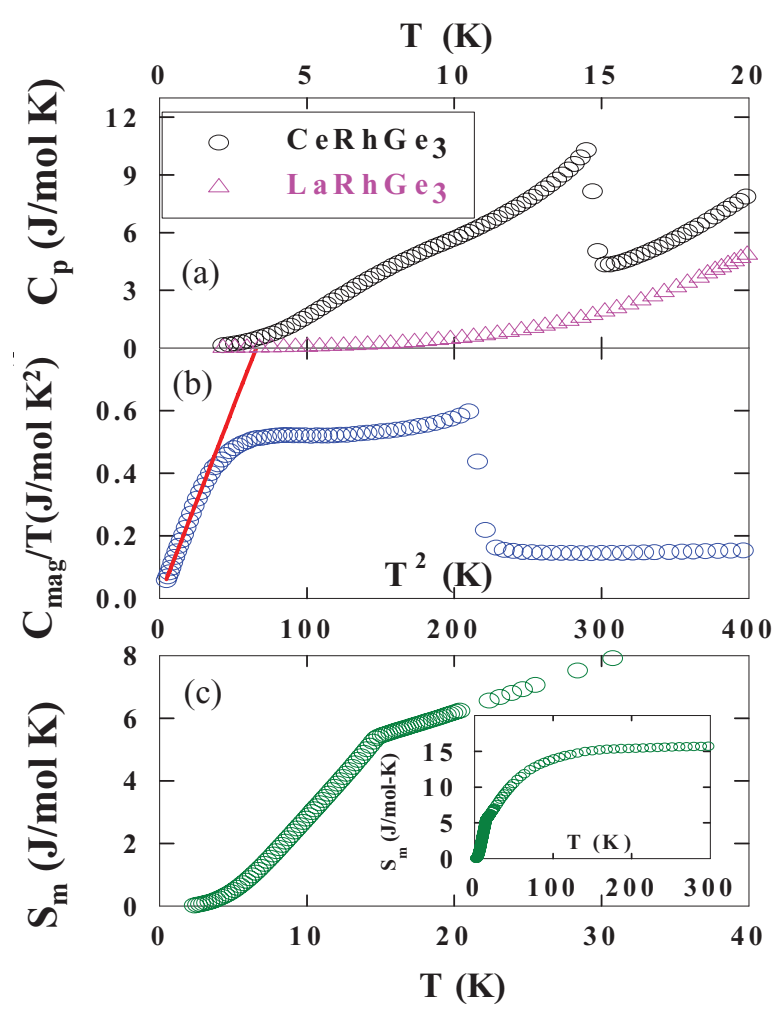

FIG. 2. (Color online) (a) Temperature dependence of the heat capacity of $\mathrm{CeRhGe}_{3}$ and the phonon reference compound $\mathrm{LaRhGe}_{3}$, (b) Estimated magnetic contribution of the heat capacity plotted as $\mathrm{C}_{\mathrm{mag}} / \mathrm{T}$ vs $\mathrm{T}^{2}$. The red solid line showing the fit (see text for details). (c) The temperature-dependent magnetic entropy estimated from the data in (b) and the inset shows the entropy up to $300 \mathrm{~K}$. 
of the Sommerfeld coefficient $\gamma \sim 5 \mathrm{~mJ} / \mathrm{mole} \mathrm{K}^{2}$ and $\beta=$ $0.415 \mathrm{~mJ} / \mathrm{mole} \mathrm{K}^{4}$. This value of $\beta$ gives a Debye temperature $\theta_{\mathrm{D}}=304 \mathrm{~K}$ for $\mathrm{LaRhGe}_{3}$. On the other hand, the heat capacity of $\mathrm{CeRhGe}_{3}$ exhibits a sharp $\lambda$-type transition at $14 \mathrm{~K}$ [Fig. 2(a)] due to the onset of AFM ordering of the Ce moments in agreement with the magnetic susceptibility. Further, a very broad peak or anomaly in the heat capacity can be seen near $7 \mathrm{~K}$ in $\mathrm{CeRhGe}_{3}$, which we suggest is due to either a change in the spin direction (or spin reorientation) of the $\mathrm{Ce}$ ions or a change in the propagation vector of the magnetic structure. The broad anomaly observed near $7 \mathrm{~K}$ is consistent with the peak observed in the magnetic susceptibility [see Fig. 1(a)], and the overall heat capacity behavior is in agreement with that reported by Muro et al. ${ }^{16}$ In order to investigate further the nature of these transitions, we also measured the heat capacity of $\mathrm{CeRhGe}_{3}$ in an applied magnetic field of $9 \mathrm{~T}$ (data not shown here) and did not detect any change within the resolution of our measurements. This is in sharp contrast to the behavior observed in a polycrystalline sample of $\mathrm{CeIrGe}_{3},{ }^{31}$ where the application of a magnetic field moves the observed sharp low-temperature transition (at $\mathrm{T}_{\mathrm{N} 2}=4 \mathrm{~K}$ ) toward a higher temperature, while it does not affect the high-temperature transition at $\mathrm{T}_{\mathrm{N} 1}=8 \mathrm{~K}$. When the applied field reaches $7 \mathrm{~T}$, the lower transition merges with the upper transition, and only one broad peak near $8 \mathrm{~K}$ can be seen in the heat capacity data. ${ }^{31} \mathrm{It}$ is very interesting to note that the application of pressure and applied magnetic field has a similar effect on the two phase transitions in $\mathrm{CeIrGe}_{3} .{ }^{17,31}$

The magnetic (or 4 f-electronic) contribution to the heat capacity of $\mathrm{CeRhGe}_{3}$ was obtained by subtracting the lattice contribution from the measured heat capacity of $\mathrm{LaRhGe}_{3}$ (i.e., by taking the difference $\mathrm{C}_{\mathrm{mag}}(\mathrm{T})=$ $\mathrm{C}_{p}\left(\mathrm{CeRhGe}_{3}\right)-\mathrm{C}_{p}\left(\mathrm{LaRhGe}_{3}\right)$. The resulting curve is plotted in Fig. 2(b) as $\mathrm{C}_{\mathrm{mag}}(\mathrm{T}) / \mathrm{T}$ vs $\mathrm{T}^{2}$. A very sharp and wellpronounced transition at $14 \mathrm{~K}$ is clearly seen in the magnetic part of the heat capacity $\mathrm{C}_{\text {mag }}(\mathrm{T})$, which further exhibits a drop near $7 \mathrm{~K}$. Although the amplitude of the change $\Delta \mathrm{C}_{\mathrm{mag}}(\mathrm{T})$ in the magnetic heat capacity, near the $14 \mathrm{~K}$ jump is similar to that of the $7 \mathrm{~K}$ drop, the jump occurs over a much narrower temperature range than the drop. A broad peak in $\mathrm{C}_{\text {mag }}$ near $40 \mathrm{~K}$ (not shown) is attributed to a crystal electric field Schottky anomaly. The magnetic contribution to the entropy was obtained by integrating the $\mathrm{C}_{\mathrm{mag}} / \mathrm{T}$ vs $\mathrm{T}$ plot and is shown in Fig. 2(c). From the temperature dependence of the magnetic entropy, we find that $S_{\text {mag }}$ attains a value of $\sim \mathrm{R} \ln 2(=5.75 \mathrm{~J} /$ mole $\mathrm{K})$ at $16 \mathrm{~K}$, suggesting a CEF-split doublet ground state in $\mathrm{CeRhGe}_{3}$. Furthermore, the entropy recovery at $300 \mathrm{~K}$ is $15.67 \mathrm{~J} /$ mole $\mathrm{K}$, which is close to $\mathrm{R} \ln 6=14.87 \mathrm{~J} /$ mole $\mathrm{K}$, indicating that the overall $\mathrm{CEF}$ splitting is smaller than $300 \mathrm{~K}$, in agreement with our INS study discussed in Sec. IIIF. We have made an estimate of the Kondo temperature $\mathrm{T}_{\mathrm{K}}$ of $\mathrm{CeRhGe}_{3}$ using the method described by Besnus et al., ${ }^{32}$ where in the mean-field approach, the jump in the heat capacity $\Delta \mathrm{C}_{\mathrm{mag}}$ of a Kondo system is related to $\mathrm{T}_{K} / \mathrm{T}_{\mathrm{N}}$. The estimate of $\mathrm{T}_{\mathrm{K}}$ through this model agrees well with the experimental data on various $\mathrm{Ce}$ and $\mathrm{Yb}$ compounds. A correlation between the specific heat jump $\Delta \mathrm{C}_{\text {mag }}$ at the magnetic ordering temperature and the ratio $\mathrm{T}_{\mathrm{K}} / \mathrm{T}_{\mathrm{N}}$ for $\mathrm{CeNi}_{x} \mathrm{Pt}_{1-x}$ has been observed by Blanco et al. ${ }^{33}$ Besnus et al. have given a universal plot of $\Delta \mathrm{C}_{\mathrm{mag}}$ vs $\mathrm{T}_{\mathrm{K}} / \mathrm{T}_{\mathrm{N}}$ for many Ce-based Kondo lattice systems. Using the observed value $\Delta \mathrm{C}_{\text {mag }}=6.44 \mathrm{~J} /$ mole $\mathrm{K}$ of $\mathrm{CeRhGe}_{3}$, we deduced $\mathrm{T}_{\mathrm{K}} / \mathrm{T}_{\mathrm{N}}=0.8$ from this universal plot that gave $\mathrm{T}_{\mathrm{K}}=11.8$ $\mathrm{K}$ for $\mathrm{CeRhGe}_{3}$. This value of $\mathrm{T}_{\mathrm{K}}$ is in reasonable agreement with that estimated from $\theta_{\mathrm{P}}$ as well as that estimated from the quasielastic linewidth discussed in Sec. IIIF. The influence of the CEF above $20 \mathrm{~K}$ and the magnetic order below $14.5 \mathrm{~K}$ makes it difficult to estimate a reliable value of the Sommerfeld coefficient $\gamma$ for $\mathrm{CeRhGe}_{3}$. An attempt has been made to estimate $\gamma$ from a linear fit to $\mathrm{C} / \mathrm{T}$ vs $\mathrm{T}^{2}$ plot above $\mathrm{T}_{\mathrm{N} 1}$ (between 15 to $20 \mathrm{~K}$ ), yielding $\gamma \sim 99 \mathrm{~mJ} / \mathrm{mole} \mathrm{K}^{2}$. However, the $\mathrm{C} / \mathrm{T}$ value at $2 \mathrm{~K}$ is only $\sim 65 \mathrm{~mJ} / \mathrm{mole} \mathrm{K}^{2}$ : this clearly reflects the influence of the $\mathrm{CEF}$ above the transition.

We now consider the spin-wave contribution to the heat capacity. From spin-wave theory, the temperature dependence of the magnetic specific heat for ferromagnets and antiferromagnets is proportional to $\mathrm{T}^{3 / 2}$ and $\mathrm{T}^{3}$, respectively, for temperatures below the ordering temperature. $\mathrm{The}_{\mathrm{mag}} / \mathrm{T}$ data of $\mathrm{CeRhGe}_{3}$ were plotted as $\mathrm{T}^{2}$ and also as $\mathrm{T}^{0.5}$, and it was found that the $\mathrm{T}^{3}$ (i.e., $\mathrm{C}_{\mathrm{mag}} / \mathrm{T}$ vs $\mathrm{T}^{2}$ plot) dependence fits better [Fig. 2(b)] below $\mathrm{T}_{\mathrm{N} 2}$, in agreement with the AFM ground state observed in $\mathrm{CeRhGe}_{3}$. For a three-dimensional (3D) antiferromagnet with a linear spin-wave dispersion relation (below $\mathrm{T}_{\mathrm{N}}$ ), the coefficient of the $\mathrm{T}^{3}$ term is given by $\mathrm{C}_{\mathrm{mag}}=$ $(8 \pi \mathrm{R} / 15)\left(\mathrm{T} / \theta_{c}\right)^{3}$, where the parameter $\theta_{c}$ is of the order of $\mathrm{T}_{\mathrm{N}} \cdot{ }^{34}$ From the measured slope, we have estimated $\theta_{c}=10.5 \mathrm{~K}$, which is indeed of the order of $\mathrm{T}_{\mathrm{N} 2}$.

\section{Muon spin relaxation}

To shed light on the two phase transitions seen in the susceptibility and heat capacity, we have investigated the temperature dependence of the muon spin relaxation in zero field. For all temperatures above the magnetic ordering temperature, the muon spin relaxation spectra can be described by a simple exponential decay. However, on cooling below the ordering temperature $\left(\mathrm{T}_{\mathrm{N} 1}=14.5 \mathrm{~K}\right)$, coherent frequency oscillations are observed in the $\mu$ SR spectra (Fig. 3). This immediately shows that the system has a long-range magnetically ordered ground state. The $\mu$ SR spectra are well described by three sinusoidal functions with a Gaussian envelope and an

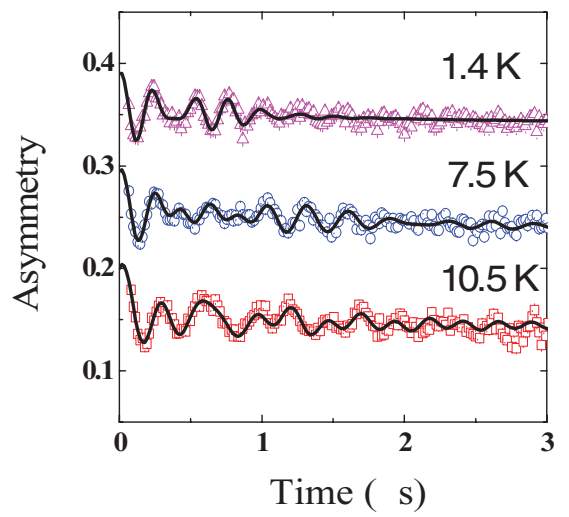

FIG. 3. (Color online) The time evolution of the muon spin relaxation for various temperatures (from top to bottom 1.4, 7.5, and $10.5 \mathrm{~K}$ ). The line is least-squares fit to the data as described in the text. Note the plots at 7.5 and $1.4 \mathrm{~K}$ are shifted vertically (by 0.1 ) for clarity. 


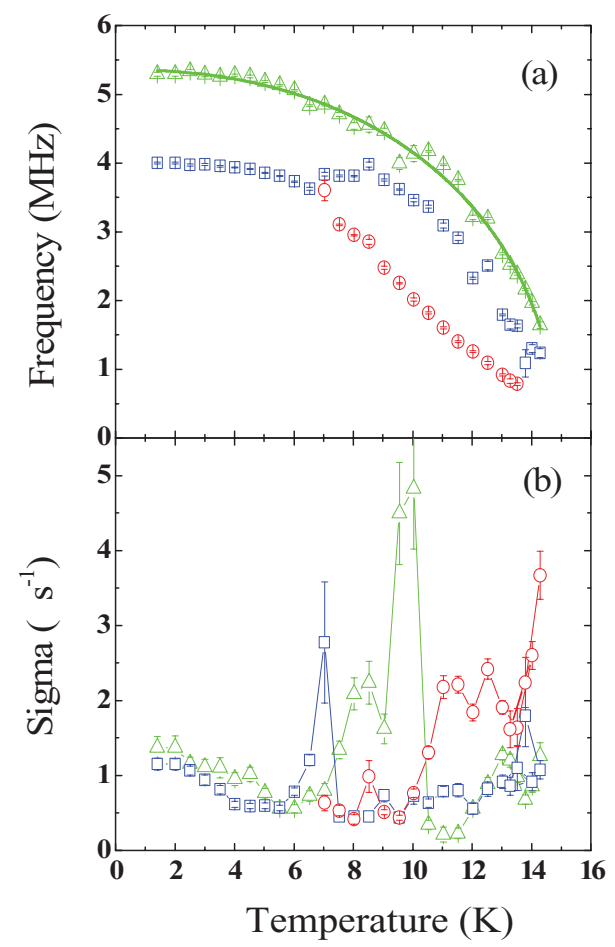

FIG. 4. (Color online) (a) The temperature dependence of the $\mu \mathrm{SR}$ frequencies for $\mathrm{CeRhGe}_{3}$. The line is a fit to the data as described in the text. (b) The temperature dependence of the Gaussian envelope $\sigma$ for $\mathrm{CeRhGe}_{3}$.

exponential decay (see Fig. 3) given by the functional form

$$
\begin{aligned}
G_{z}(t)= & \left(\sum_{i=1}^{3} A_{i} \cos \left(2 \pi v_{i} t+\varphi\right) \exp \left(-\frac{\left(\sigma_{i} \mathrm{t}\right)^{2}}{2}\right)\right) \\
& +A_{0} \exp (-\lambda \mathrm{t})+\mathrm{A}_{\text {background }},
\end{aligned}
$$

where $\mathrm{A}_{i}\left(\mathrm{~A}_{0}\right)$ is the initial asymmetry, $\sigma_{i}$ is the Gaussian envelope to the oscillating function with a frequency of $v_{i}$ and a phase $\varphi, \lambda$ is the muon spin relaxation rate, and $\mathrm{A}_{\text {background }}$ is the background. Although one might expect the addition of three exponential relaxation functions, the data suggest that these values of $\lambda$ would be so close that they can be fitted with one value.

Next, we consider the temperature dependence of the muon frequencies below $\mathrm{T}_{\mathrm{N} 1}$ shown in Fig. 4(a). There are three distinct frequencies for temperatures greater than $\sim 7 \mathrm{~K}$. However, as the temperature is reduced below $7 \mathrm{~K}$, the middle frequency merges into the lowest frequency. Normally, three frequencies imply three distinct muon sites, but considering the crystal structure of $\mathrm{CeRhGe}_{3}$, it is not obvious where these three sites could be. Interestingly, as discussed in Sec. III E, the magnetic structure of $\mathrm{CeRhGe}_{3}$ is a spin-density wave type (see Fig. 6), and hence there are two types of crystallographic unit cells in the magnetic unit cell at $2 \mathrm{~K}$. In one cell the Ce-layer (made up of four Ce atoms) has a $\mathrm{z}$-component of the magnetic moment [see Fig. 6(a) bottom unit cell], while in the next cell this Ce layer does not have a z-component of the magnetic moment [zero moment, see Fig. 6(a) or smaller moment, see Fig. 6(b) second unit cell from the bottom]. This can explain the observed two frequencies below $7 \mathrm{~K}$. Intriguingly, the presence of the three frequencies above $7 \mathrm{~K}$ indicates that there is clearly a change in the magnetic structure with temperature, i.e., we have two different magnetic structures below and above $7 \mathrm{~K}$. It suggests that we have a complex magnetic structure also above $7 \mathrm{~K}$, which is likely to be incommensurate with the lattice.

The temperature dependence of the upper frequency, which is a measure of the internal field at the muon site, can be fitted with

$$
v(T)=v(0)\left(1-\left(\frac{T}{T_{N}}\right)^{\alpha}\right)^{\beta}
$$

The results of the fit gives $v(0)=5.40(6) \mathrm{MHz}, \mathrm{T}_{\mathrm{N}}=$ 14.8(2) $\mathrm{K}, \alpha=2.3(2)$ and $\beta=0.48(7)$, which being close to $\frac{1}{2}$ indicates that $\mathrm{CeRhGe}_{3}$ can be thought of as a mean-field magnet. The value of $\alpha$, which is much greater than 1 , implies that there are complex interactions between the moments as inferred through our neutron diffraction study in Sec. III E that reveals a complex spin arrangement in the magnetic unit cell.

Finally, the temperature dependence of $\sigma$ is shown in Fig. 4(b). Near the temperature at which we observe the three frequencies merging into two, we also see a peak in $\sigma(\mathrm{T})$. Further, the $\sigma(\mathrm{T})$ also exhibits a peak near $10 \mathrm{~K}$, where the highest frequency exhibits a weak anomaly. The peaks in the $\sigma(\mathrm{T})$ indicate an increase in the distribution of the internal fields at the muon site(s) around the main field. Each peak shows that either an increase in the spin fluctuations or a very small change in the magnetic structure occurs, possibly a small

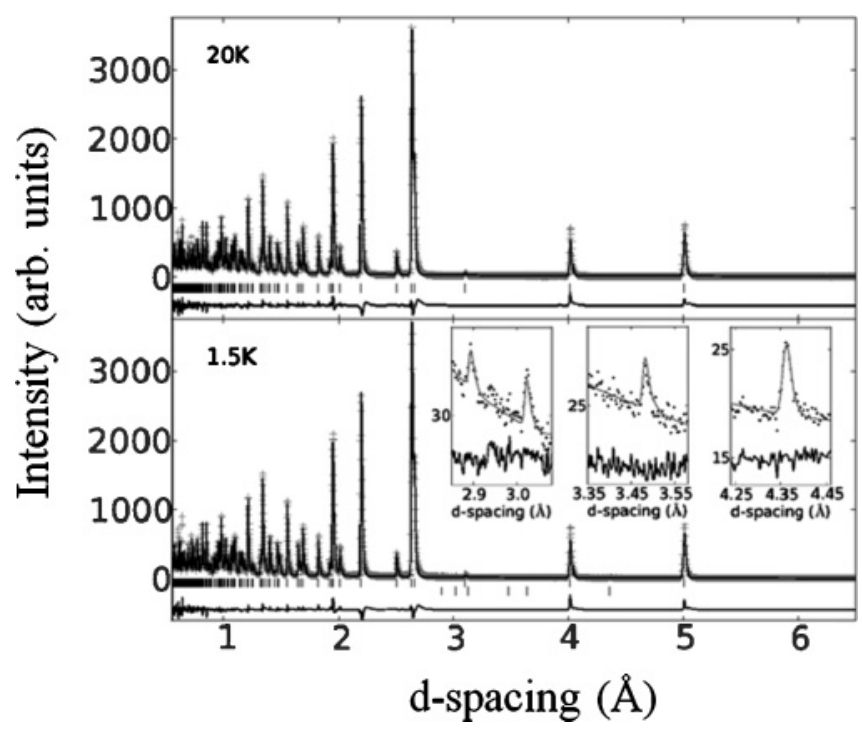

FIG. 5. Rietveld refinement of the neutron powder diffraction pattern (obtained from WISH) of $\mathrm{CeRhGe}_{3}$ at $20 \mathrm{~K}$ (top) and $1.5 \mathrm{~K}$ (bottom). The data are shown as grey crosses, and the result of the refinement as a solid (black) line. The upper and lower rows of tick marks indicate the position of nuclear and magnetic Bragg peaks, respectively. The difference curve between the experimental data and the refinement is shown at the bottom of both figures. The insets in the bottom figure display an enlarged region of the diffraction pattern near the magnetic Bragg peaks along with the refinement. 
change in the modulation wave vector $(\mathbf{k})$ of the magnetic moment, within the $\mu \mathrm{SR}$ time window.

\section{E. Magnetic neutron diffraction}

Figure 5 shows the neutron diffraction data collected at 20 and $1.5 \mathrm{~K}$ for the $90^{\circ}$ detector bank on WISH. The refinement (weighted profile factor $\mathrm{R}_{\mathrm{wp}}=5.34 \%$ ) at $20 \mathrm{~K}$ confirms that $\mathrm{CeRhGe}_{3}$ crystallizes in the tetragonal space group $14 \mathrm{~mm}$, in agreement with both our GEM data and the previous report, ${ }^{17}$ with $\mathrm{a}=4.385(2)$ and $\mathrm{c}=10.015(3) \AA$ at $20 \mathrm{~K}$. The presence of a small impurity, which could not be indexed by any of the binary and tertiary compounds of $\mathrm{Ce}-\mathrm{Rh}-\mathrm{Ge}$ reported in the ICSD database ${ }^{35}$ was also detected. At $1.5 \mathrm{~K}$, the diffraction data reveals the presence of four clear additional peaks at 4.36, 3.48, 3.02, and $2.89 \AA$ (insets of Fig. 5), and two possible very weak peaks at 2.17 and $1.8 \AA$ that are better resolved in the higher-angle detector banks (not shown). The intensity of these peaks decreases with the modulus of the scattering vector (Q) as expected from the Q-dependence of the magnetic form factor. It is to be noted that only $\mathrm{Ce}$ atoms have magnetic moment ( $\mathrm{Rh}$ and Ge atoms do not carry magnetic moment), and there is only one crystallographic site for the Ce atoms per magnetic unit cell and other Ce atoms are related by the symmetry operators. Further evidence of the magnetic origin of these extra peaks is provided by the reduction of the background at low $\mathrm{Q}$. This is the expected behavior at a magnetic transition where the intensity of the paramagnetic scattering is transferred to the Bragg scattering. Initially, an automatic indexing procedure using a grid search was used to determine the periodicity of the magnetic structure. To confine the wave vector search, all the special points and lines of the Brillouin zone generated by the program ISOTROPY ${ }^{36}$ were tested. The best solution, accounting for all the observed peaks, was found along the line $\operatorname{LD}(0,0,2 \mathrm{a})$ for the wave vector $\mathbf{k}=(0,0,3 / 4)$. This wave vector corresponds to $(3 / 8,3 / 8,-3 / 8)$ in the primitive cell and can be labeled $\mathrm{k}_{10}$ in Kovalev's ${ }^{37}$ notation. At this point, it is worth mentioning that the same wave vector $\mathbf{k}=(0,0,3 / 4)$ has also been observed in a recent single-crystal neutron diffraction study by Kaneko et al. ${ }^{21}$ on isostructural $\mathrm{CeCoGe}_{3}$. However, these authors also reveal the existence of another dominant wave vector $\mathbf{k}^{\prime}=(0,0,1 / 2)$, not detected in our $\mathrm{CeRhGe}_{3}$ sample, with intensities associated with $\mathbf{k}^{\prime}$ being a factor 7 higher than those associated with $\mathbf{k}=(0,0,3 / 4)$. None of the other special points of the Brillouin zone, nor a full 3D grid search, gave a better R-factor. Symmetry analysis indicates that there are four, one-dimensional (1D) representations, labeled $\Gamma_{1}$ to $\Gamma_{4}$ and one, two-dimensional (2D) representation $\Gamma_{5}$ in the little group (group of the propagation vector), although only $\Gamma_{2}$ and $\Gamma_{5}$ enter the decomposition of $\Gamma_{\text {mag }}=\Gamma_{2}+\Gamma_{5}$. $\Gamma_{2}$ and $\Gamma_{5}$ correspond to an ordering of the Ce sites along the $c$ axis and in the ab-plane, respectively. A good fit to the data (magnetic Bragg factor $\mathrm{R}_{\mathrm{B}}=11.9 \%$ ) was obtained using FullProf ${ }^{38}$ with the representation $\Gamma_{2}$. Attempts at refinements using $\Gamma_{5}$ gave an $R_{B}$ a factor 6 to 7 times higher with the peaks at $2.89,3.02$, and $4.36 \AA$ being fairly well accounted for, but the one at $3.48 \AA$ overestimated. Using $\Gamma_{5}$ also introduces some intensity in the $(002)+\mathbf{k}$ peak at $3.64 \AA$, which is not very clear in the $90^{\circ}$ bank data and (a)

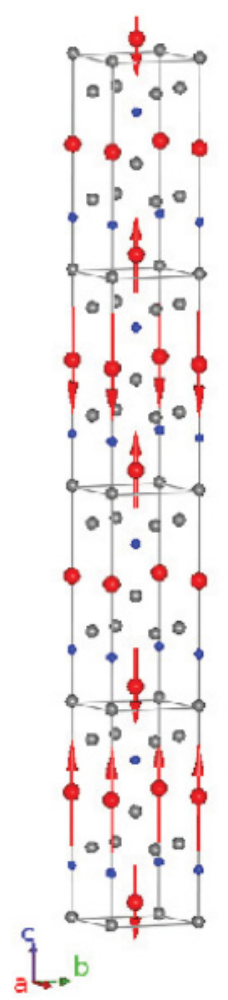

(b)

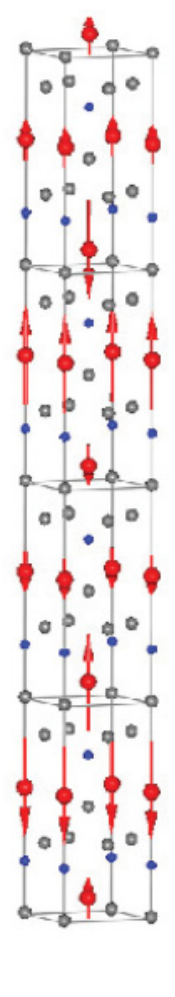

FIG. 6. (Color online) Magnetic unit cell of $\mathrm{CeRhGe}_{3}$ (a) with zero value of the global phase and (b) with the global phase of $9 / 16$ $* \pi \bmod (\pi / 4)$. The large red (dark gray), small blue (medium gray), and large gray symbols indicate $\mathrm{Ce}, \mathrm{Rh}$, and $\mathrm{Ge}$ atoms, respectively. The red arrows show the direction and size of the Ce magnetic moment.

also in the (002)-k peak at $8.02 \AA$ is not visible in the lower-angle diffraction bank. The refined moment, using the $\Gamma_{2}$ representation, was $0.45(9) \mu_{\mathrm{B}}$, and the magnetic structure is depicted in Fig. 6. It should be noted that the moment direction obtained from our neutron diffraction study at $1.5 \mathrm{~K}$ is along the $c$ axis, which is in agreement with the drop in the $c$ axis susceptibility. ${ }^{17}$ On the other hand, the easy axis (i.e., the axis with the highest magnetization in applied magnetic field) observed in the antiferromagnetic (AFM) state from the magnetization data at $2 \mathrm{~K}$ is the $a$ axis (see Fig. 5 in Ref. 17) and not the $c$ axis. Further, the observed moment along the $c$ axis in $\mathrm{CeRhGe}_{3}$ is also in contrast with the highest susceptibility direction, which is the $a$ axis for $\mathrm{T}>\mathrm{T}_{\mathrm{N}} .{ }^{17}$

It should be noted that neutron diffraction patterns obtained from a given magnetic structure are identical when a global phase $\Phi$ is added. Figure 6(a) shows the magnetic structure obtained for a $\Phi$ equals to zero and includes some $\mathrm{Ce}$ sites having zero moment. In the present case, no suitable global phase that would result in a constant moment solution could be found but a special case when the global phase $=$ $9 / 16^{*} \pi \bmod (\pi / 4)=0.5625^{*} \pi \bmod (\pi / 4)$ [see Fig. 6(b)] for which the values of the moments on the first $\mathrm{Ce} 1$ site at $(00$ $0.579)$ are equal the ones on the second $\mathrm{Ce} 2$ site at $(0.50 .5$ 0.579) (still the moments are not constant on either of the sites and are along the $c$ axis), i.e., Ce1 atoms translated by $\left(\begin{array}{ll}0 & 0 \mathrm{n}\end{array}\right)$ 


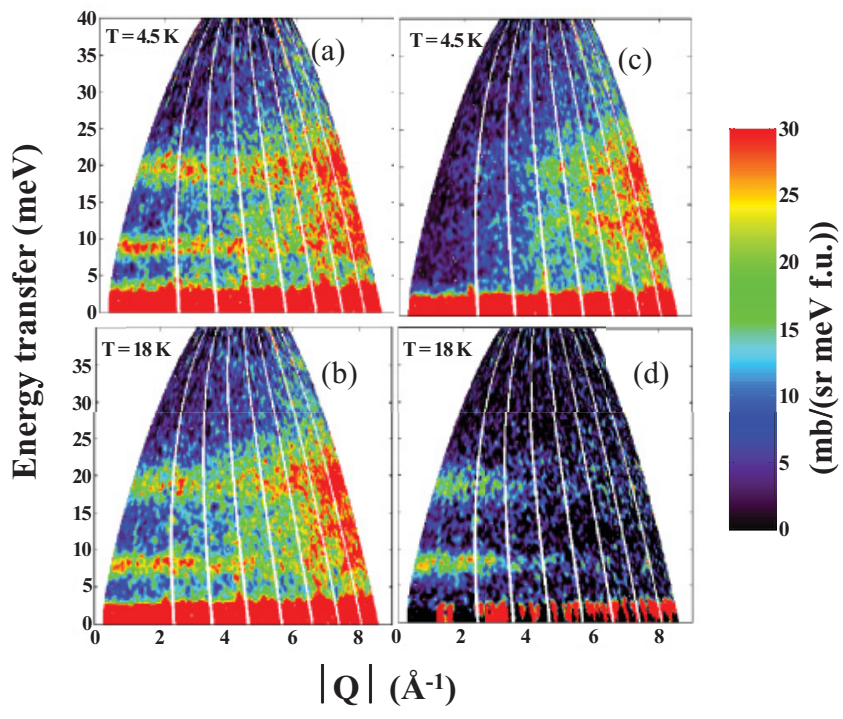

FIG. 7. (Color online) Contour plots of the inelastic scattering (in $\mathrm{mb} / \mathrm{sr} \mathrm{meV}$ f.u.) with energy transfer (E) vs wave vector transfer (Q), (a) and (b) of $\mathrm{CeRhGe}_{3}$ at 4.5 and $18 \mathrm{~K}$ and (c) of $\mathrm{LaRhGe}_{3}$ at $4.5 \mathrm{~K}$. (d) The magnetic scattering of $\mathrm{CeRhGe}_{3}$ at $18 \mathrm{~K}$ estimated by subtracting the data of $\mathrm{LaRhGe}_{3}$.

( $\mathrm{n}=0,1,2,3)$ have a moment of $\mu_{\mathrm{z}}=-0.42,-0.17,0.42$, $0.17 \mu_{\mathrm{B}}$, respectively, and $\mathrm{Ce} 2$ atoms translated by $(00 \mathrm{n})$ have a moment of $\mu_{\mathrm{z}}=0.17,0.42,-0.17,-0.42 \mu_{\mathrm{B}}$, respectively. As we are unable to determine the muon site in $\mathrm{CeRhGe}_{3}$ from our present $\mu \mathrm{SR}$ study on the polycrystalline sample, single-crystal nuclear magnetic resonance (NMR) and $\mu \mathrm{SR}$ studies should prove very useful to determine the unique value of the global phase of the magnetic structure of $\mathrm{CeRhGe}_{3}$.

\section{F. Inelastic neutron scattering}

\section{High-energy INS study to investigate CEF excitations}

Figures 7(a) and 7(b) show contour plots of the INS, measured on the MARI spectrometer with $\mathrm{E}_{i}=45 \mathrm{meV}$, from $\mathrm{CeRhGe}_{3}$ at 4.5 and $18 \mathrm{~K}$ and also from the phonon reference compound $\mathrm{LaRhGe}_{3}$ at $4.5 \mathrm{~K}$ [Fig. 7(c)]. In the paramagnetic state (at $18 \mathrm{~K}>\mathrm{T}_{\mathrm{N}}$ ) at low scattering vector $(\mathrm{Q})$, two inelastic excitations near 7.5 and $18 \mathrm{meV}$ can be clearly seen in $\mathrm{CeRhGe}_{3}$. Similar scattering has also been observed at 4.5 and $10 \mathrm{~K}$, which indicates that scattering from the spin waves in the ordered state of $\mathrm{CeRhGe}_{3}$ is at very low energy below $3.5 \mathrm{meV}$ and is confirmed by our low-energy study discussed in Sec. III F2. In contrast the scattering in $\mathrm{LaRhGe}_{3}$ is very weak at low Q [Fig. 7(c)] but exhibits at high Q the same two peak structure, as seen in $\mathrm{CeRhGe}_{3}$, with nearly similar intensity. This shows the presence of two magnetic excitations in $\mathrm{CeRhGe}_{3}$ having similar energies to the phonon peaks. It is interesting to mention that despite having similar energy scales, the magnetic and phonon excitations appear uncoupled and do not show any CEF-phonon coupling effect, which would give rise to an extra magnetic-CEF peak over the number expected from Kramers theorem. ${ }^{39}$ According to this theorem, one cannot observe more than two $\mathrm{CEF}$ excitations from the $\mathrm{J}=5 / 2$ ground state of $\mathrm{Ce}^{3+}$ ion in any Ce compound in the paramagnetic state. Such CEF-phonon coupling has been

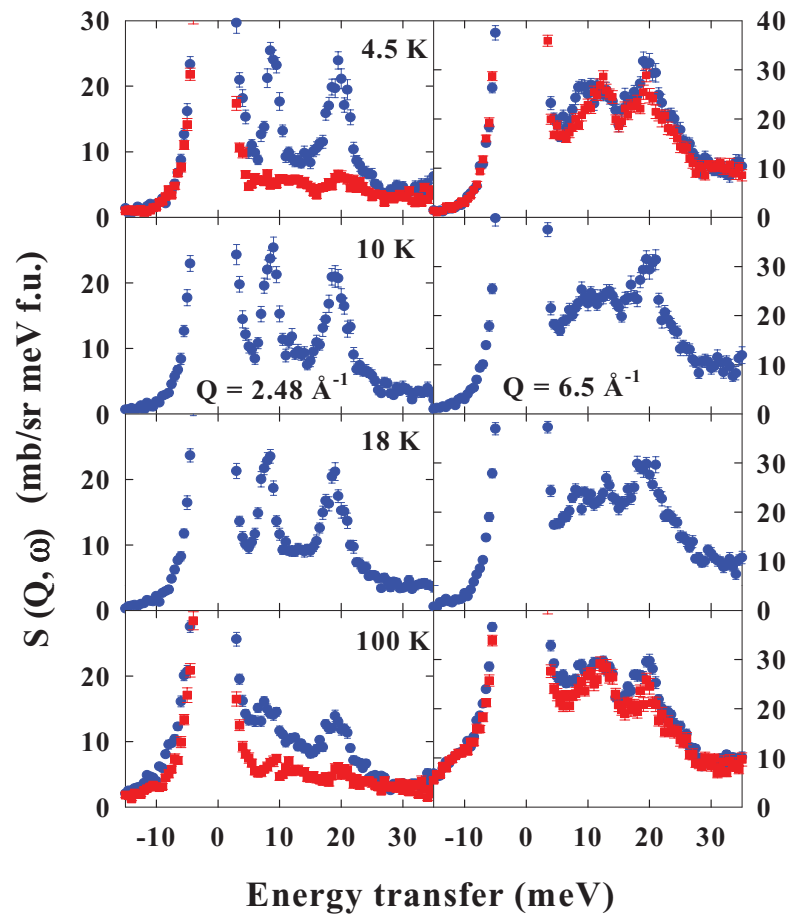

FIG. 8. (Color online) The Q-integrated 1D cuts of the total scattering from $\mathrm{CeRhGe}_{3}$ (blue circles) and $\mathrm{LaRhGe}_{3}$ (red squares) at low $Q=0$ to $4 \AA^{-1}$ (left side) and at high $Q=5$ to $9 \AA^{-1}$ (right side).

detected in $\mathrm{CeCuAl}_{3}$, which has a similar crystal structure to $\mathrm{CeRhGe}_{3}$, where three CEF excitations from the ground state are clearly observed. ${ }^{40}$

The scattering at the highest $\mathrm{Q}$ is comparable for $\mathrm{CeRhGe}_{3}$ and $\mathrm{LaRhGe}_{3}$, which indicates similar phonon contributions in these compounds. This can be seen clearly in the 1D cuts made from the $2 \mathrm{D}$ color plots at low $\mathrm{Q}$ from 0 to $4 \AA^{-1}$ and at high Q from 5 to $9 \AA^{-1}$ (see Fig. 8) at 4.5, 10, 18, and $100 \mathrm{~K}$. At 4.5 and $100 \mathrm{~K}$ the phonon contributions at high $\mathrm{Q}$ are the same in both compounds, but at low Q, the magnetic scattering is strong and with a small phonon contribution in $\mathrm{CeRhGe}_{3}$. The magnetic scattering has been deduced by subtracting the observed scattering for $\mathrm{LaRhGe}_{3}$ from that of $\mathrm{CeRhGe}_{3}$, i.e., $\mathrm{S}_{\mathrm{M}}(\mathrm{Q}, \omega)=\mathrm{S}\left(\mathrm{Q}, \omega, \mathrm{CeRhGe}_{3}\right)-\mathrm{S}\left(\mathrm{Q}, \omega, \mathrm{LaRhGe}_{3}\right)$. This is shown in Fig. 7(d), which clearly reveals the presence of two excitations. The Q-integrated (from 0 to $4 \AA^{-1}$ ) $1 \mathrm{D}$ cuts of the magnetic scattering are shown in Fig. 9 at all four temperatures. Figure 9(c) shows two well-resolved inelastic excitations at $18 \mathrm{~K}$ (i.e., above $\mathrm{T}_{\mathrm{N}}$ ), which we attribute to the CEF excitations from the ground-state CEF level to two excited CEF levels: the $\mathrm{J}=5 / 2$ ground-state multiplet of the $\mathrm{Ce}^{3+}$ ion in $\mathrm{CeRhGe}_{3}$ splits into three CEF doublets and hence gives two CEF excitations. To check the Q-dependence of the intensity of these excitations, we have plotted the energy integrated intensity between 6 and $9 \mathrm{meV}$ and between 16 and $21 \mathrm{meV}$, as a function of $\mathrm{Q}$ in Fig. 10. The intensity of both excitations decreases with increasing $\mathrm{Q}$, following the square of the magnetic form factor $F^{2}(Q)$ for a $\mathrm{Ce}^{3+}$ ion. A similar Q-dependence was also observed at $4.5 \mathrm{~K}$. As 


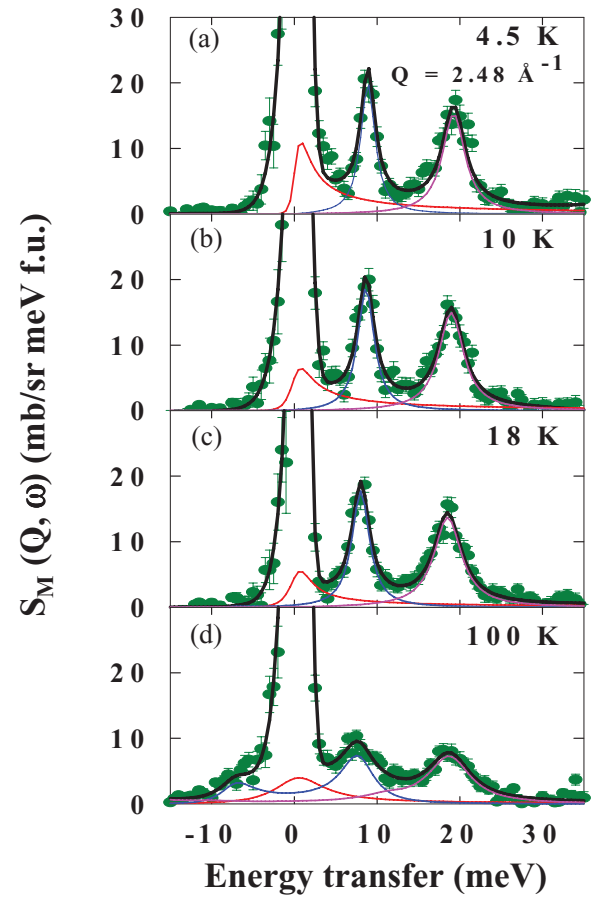

FIG. 9. (Color online) The estimated magnetic scattering from $\mathrm{CeRhGe}_{3}$ at low $\mathrm{Q}=0$ to $4 \AA^{-1}$ at $4.5,10,18$, and $100 \mathrm{~K}$. The thick solid lines represent the fit (based on the CEF model), and thin solid lines show the components of the fit (see text).

expected, the phonon scattering increases with $\mathrm{Q}$ and follows a $\mathrm{Q}^{2}$ dependence.

On further decreasing the temperature to 10 and $4.5 \mathrm{~K}$, a small increase in the energy of the CEF excitations can be noticed compared to the values at $18 \mathrm{~K}$, which is due to either the presence of a molecular field in the magnetically ordered state or the possibility of magnetostriction below $\mathrm{T}_{\mathrm{N}}$. At $18 \mathrm{~K}$ the linewidth of the $7.5 \mathrm{meV}$ excitation is $1.4(2) \mathrm{meV}$, while that of $18 \mathrm{meV}$ excitation is $2.2(3) \mathrm{meV}$ for $\mathrm{CeRhGe}_{3}$. This latter value is significantly greater than the instrumental resolution of $1.0 \mathrm{meV}$ for this energy transfer. Considering that $\mathrm{CeRhGe}_{3}$ is a crystallographically ordered system with one Ce site, the larger linewidth of the higher-energy excitation, compared to the resolution, may be attributed to the presence of a strong hybridization between the wave functions of this particular CEF level with the conduction electrons, indicating the presence of anisotropic hybridization. It is interesting to compare the energy scale and linewidths of the CEF excitations in $\mathrm{CeRhGe}_{3}$, with those of $\mathrm{CeRhSi}_{3}$, which has same crystal structure but shows pressure-induced SC with $\mathrm{T}_{\mathrm{sc}}=1 \mathrm{~K}$ at $2.5 \mathrm{GPa}$. The INS study on $\mathrm{CeRhSi}_{3}$ shows two CEF excitations at 19 and $36 \mathrm{meV}$ with linewidths of 3.9(2) and 9.2(4) meV, respectively. ${ }^{7}$ The overall CEF splitting and linewidths in $\mathrm{CeRhSi}_{3}$ are higher than those observed in $\mathrm{CeRhGe}_{3}$, which indicates stronger hybridization for the $\mathrm{Si}$ compound. The weaker hybridization observed in $\mathrm{CeRhGe}_{3}$ compared with that in $\mathrm{CeRhSi}_{3}$ through our INS study is also in agreement with that proposed by Kawai et al. ${ }^{17}$ based on the molar volume vs $\mathrm{T}_{\mathrm{N}}$ and molar volume vs $\gamma$ plots. In these plots, $\mathrm{CeRhSi}_{3}$ is close to a $\mathrm{QCP}$, while $\mathrm{CeRhGe}_{3}$ is well below the QCP (on the localized limit side with

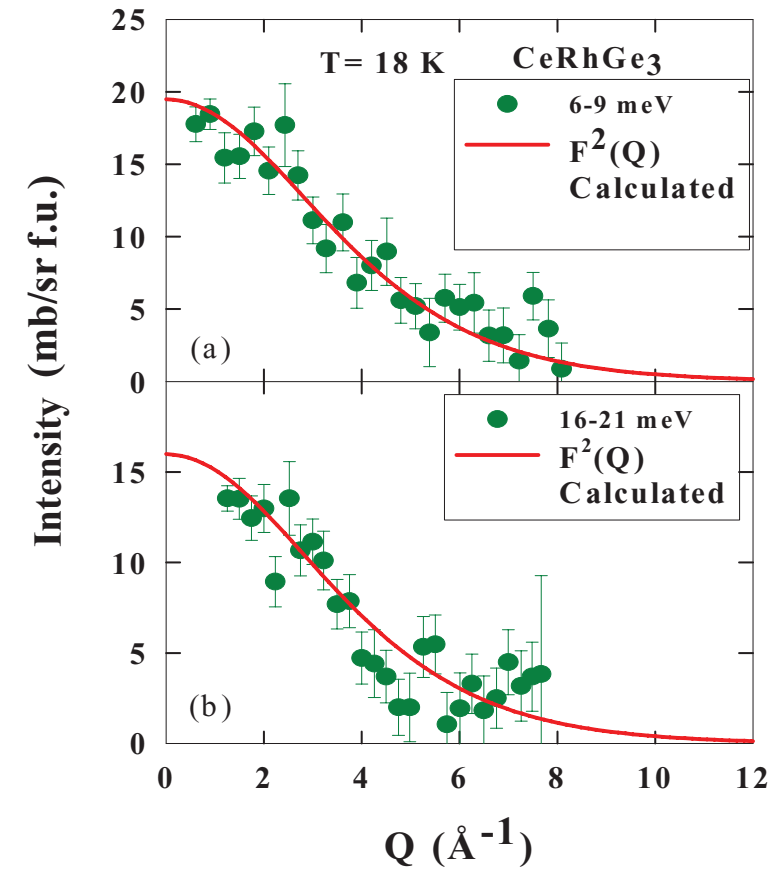

FIG. 10. (Color online) The energy integrated [from 6-9 meV (a) and 16-21 meV (b)] Q-dependent magnetic scattering from $\mathrm{CeRhGe}_{3}$ at $18 \mathrm{~K}$. The solid line represents the $\mathrm{Ce}^{3+}$ magnetic form factor squared calculated from P. J. Brown's tables (Ref. 53).

smaller hybridization). The observation of well-resolved CEF excitations in $\mathrm{CeRhGe}_{3}$ supports Kawai's analysis.

We now proceed with a more detailed analysis of the observed CEF excitations. In the tetragonal point symmetry, $4 \mathrm{~mm}\left(\mathrm{C}_{4 \mathrm{v}}\right)$, at the Ce site, the CEF Hamiltonian can be represented as follows:

$$
H_{C F}=B_{2}^{0} O_{2}^{0}+B_{4}^{0} O_{4}^{0}+B_{4}^{4} O_{4}^{4},
$$

where $\mathrm{B}_{\mathrm{n}}^{\mathrm{m}}$ are the CEF parameters to be determined from the experimental data and $\mathrm{O}_{\mathrm{n}}^{\mathrm{m}}$ are Stevens operator equivalents obtained using the angular momentum operators. ${ }^{41}$ The value of $\mathrm{B}_{2}^{0}$ can be accurately determined using the high-temperature expansion of the magnetic susceptibility, ${ }^{42}$ which gives $\mathrm{B}_{2}^{0}$ in terms of the $\mathrm{CW}$ temperatures, $\theta_{\mathrm{ab}}$ for an applied field in the ab-plane and $\theta_{c}$ for an applied field parallel to $c$ axis:

$$
B_{2}^{0}=\frac{10}{3}\left(\theta_{a b}-\theta_{c}\right) /(2 J-1)(2 J+3) .
$$

Note that Eq. (5) is valid for isotropic exchange interactions (i.e., isotropic molecular field parameters). Using the values of $\theta_{\mathrm{ab}}$ and $\theta_{c}$ from the single-crystal susceptibility, ${ }^{17}$ we find $\mathrm{B}_{2}^{0}=0.206 \mathrm{meV}$ from the high-temperature $\mathrm{CW}$ behavior between 150 and $300 \mathrm{~K}$ and $\mathrm{B}_{2}^{0}=0.135 \mathrm{meV}$ from the low-temperature $\mathrm{CW}$ behavior between 30 and $135 \mathrm{~K}$. In our subsequent analysis, we first kept the value of $\mathrm{B}_{2}^{0}$ (both values were tried) from this estimate fixed and then varied $\mathrm{B}_{4}^{0}$ and $\mathrm{B}_{4}^{4}$. Although we could get a good fit to the neutron data at $18 \mathrm{~K}$ and $100 \mathrm{~K}$ with these values of $\mathrm{B}_{2}^{0}$, the estimated CEF parameters did not give a good fit to the single-crystal susceptibility data for the entire temperature range from $\mathrm{T}>\mathrm{T}_{\mathrm{N}}$ to $300 \mathrm{~K}$. Hence in the final fit, we also allowed $\mathrm{B}_{2}^{0}$ to vary and fitted the neutron scattering data (at each temperature) together with the single-crystal susceptibility. The values of the parameters 
TABLE III. CEF parameters $\mathrm{B}_{\mathrm{n}}^{\mathrm{m}}$, molecular field parameters $\left(\lambda^{\xi}, \xi=\perp\right.$ and $\|$ to $c$ axis) and temperature-independent constant susceptibility $\left(\chi_{0}^{\xi}\right)$, calculated CEF ground-state magnetic moments $\left(\left\langle\mu_{x}\right\rangle\right.$ and $\left\langle\mu_{z}\right\rangle$ calculated from the CEF ground-state wave functions) and the calculated energy ( $\Delta \mathrm{E}_{1}$ and $\Delta \mathrm{E}_{2}$ are the energy of first- and second-excited CEF levels) of the CEF levels. The parameters were estimated by simultaneous fit to the inelastic neutron data and the single-crystal susceptibility at each temperature.

\begin{tabular}{|c|c|c|c|c|}
\hline & $4.5 \mathrm{~K}$ & $10 \mathrm{~K}$ & $18 \mathrm{~K}$ & $100 \mathrm{~K}$ \\
\hline $\mathrm{B}_{2}^{0}(\mathrm{meV})$ & 0.381 & 0.385 & 0.393 & 0.408 \\
\hline $\mathrm{B}_{4}^{0}(\mathrm{meV})$ & 0.021 & 0.021 & 0.018 & 0.018 \\
\hline $\mathrm{B}_{4}^{4}(\mathrm{meV})$ & 0.308 & 0.302 & 0.294 & 0.301 \\
\hline$\lambda^{\perp}(\mathrm{mole} / \mathrm{emu})$ & -17.286 & -17.586 & -19.787 & -19.919 \\
\hline$\lambda^{\|}(\mathrm{mole} / \mathrm{emu})$ & 10.939 & 8.671 & 8.079 & 10.396 \\
\hline $\begin{array}{l}\chi_{0}^{\perp} \\
\left(\times 10^{-3} \mathrm{emu} / \mathrm{mol}\right)\end{array}$ & -1.086 & -1.090 & -1.091 & -1.094 \\
\hline $\begin{array}{l}\chi_{0}^{\|} \\
\left(\times 10^{-3} \mathrm{emu} / \mathrm{mol}\right)\end{array}$ & -0.964 & -0.948 & -0.940 & -0.931 \\
\hline$\left\langle\mu_{\mathrm{x}}\right\rangle\left(\mu_{\mathrm{B}}\right)$ & 0.83 & 0.83 & 0.83 & 0.83 \\
\hline$\left\langle\mu_{\mathrm{z}}\right\rangle\left(\mu_{\mathrm{B}}\right)$ & 0.43 & 0.44 & 0.43 & 0.42 \\
\hline$\Delta \mathrm{E}_{1}(\mathrm{meV})$ & 8.73 & 8.53 & 7.66 & 7.62 \\
\hline$\Delta \mathrm{E}_{2}(\mathrm{meV})$ & 19.10 & 18.84 & 18.20 & 18.58 \\
\hline
\end{tabular}

obtained from this procedure are given in Table III, and the quality of the fit to the measured data can be seen in Fig. 1(b) for the susceptibility and Fig. 9 for the neutron scattering data. The estimated value of $\mathrm{B}_{2}^{0}$ is higher than that found from the susceptibility, while $\mathrm{B}_{4}^{0}$ is very weak, and $\mathrm{B}_{4}^{4}$ is the second largest parameter. The CEF wave functions obtained from the simultaneous fit to $18 \mathrm{~K}$ INS and the susceptibility data are given by

$$
\begin{gathered}
\Psi_{1}^{ \pm}=(0.8658)\left| \pm \frac{3}{2}\right\rangle-(0.5004)\left|\mp \frac{5}{2}\right\rangle \\
\Psi_{2}^{ \pm}=\left| \pm \frac{1}{2}\right\rangle \\
\Psi_{3}^{ \pm}=(0.8658)\left| \pm \frac{5}{2}\right\rangle+(0.5004)\left|\mp \frac{3}{2}\right\rangle
\end{gathered}
$$

They correspond to the energy eigenvalues of $0,7.7$, and $18.2 \mathrm{meV}$. We can now estimate the value of the ground state magnetic moment using the above wave functions and the following formulae:

$$
\begin{aligned}
& \left\langle\mu_{x}\right\rangle=\left\langle\Psi_{1}^{ \pm}\left|\frac{g_{J}}{2}\left(J^{+}+J^{-}\right)\right| \Psi_{1}^{\mp}\right\rangle \\
& \left\langle\mu_{z}\right\rangle=\left\langle\Psi_{1}^{ \pm}\left|g_{J}\left(J_{Z}\right)\right| \Psi_{1}^{ \pm}\right\rangle .
\end{aligned}
$$

The estimated magnetic moments $\left\langle\mu_{x}\right\rangle$ and $\left\langle\mu_{z}\right\rangle$ are given in Table III. The value of $\left\langle\mu_{z}\right\rangle$ estimated here is in excellent agreement with that obtained from the neutron diffraction study. However, on the basis of the CEF model one would expect the ordered state $\mathrm{Ce}$ moment in the ab-plane and not along the $c$ axis. Now we consider the direction of the moment. According to the single-crystal susceptibility data in the paramagnetic state and also from the analysis of our INS data, $\mathrm{B}_{2}^{0}$ is positive. If we consider the magnetocrystalline anisotropy energy in terms of the second-order CEF parameter, which is given, in the lowest order term in the energy by ${ }^{43}$

$$
E_{a}=K_{1} \sin ^{2}(\theta) .
$$

Here $\theta$ is the angle between the moment direction and the $\left[\begin{array}{lll}0 & 0 & 1\end{array}\right]$ axis. If $K_{1}>0$, the directions of lowest energy are the $\pm z$ directions, and $z$ axis is the easy axis. If $K_{1}<0$, there is an easy plane perpendicular to the symmetry axis (the basal plane of the crystal). Hence from the sign of $\mathrm{B}_{2}^{0}$ one can predict the magnetization direction. For $\mathrm{Ce}^{3+}$ systems a positive $\mathrm{B}_{2}^{0}\left(\mathrm{~K}_{1}<0\right)$ minimizes $\mathrm{E}_{\mathrm{a}}$ when the angle $\theta$ is $90^{\circ}$, i.e., moment in the ab-plane, while for negative $B_{2}^{0}\left(K_{1}>\right.$ $0), \mathrm{E}_{\mathrm{a}}$ will be minimum for the moment along the $c$ axis. In $\mathrm{CeRhGe}_{3}$ we have a positive $\mathrm{B}_{2}^{0}$, which predicts that the moment should be in the ab-plane. But the observed moment at $1.5 \mathrm{~K}$ is along the $c$ axis. This remains the case even when we include the $\mathrm{B}_{4}^{4}$ term (the weak $\mathrm{B}_{4}^{0}$ can be neglected) in the anisotropy energy. This suggests that an anisotropy other than the magnetocrystalline anisotropy is playing an important role in determining the direction of the moments in $\mathrm{CeRhGe}_{3}$. We attribute this anomaly of the magnetic moment direction in $\mathrm{CeRhGe}_{3}$ to the presence of two-ion anisotropic magnetic exchange interactions between the $\mathrm{Ce}$ ions, along the $c$ axis and in the ab-plane. ${ }^{44}$ This interpretation has been supported through the estimated anisotropic molecular field parameters (see Table III) from the susceptibility analysis, negative sign for $\lambda^{\perp}$ and positive sign for $\lambda^{\|}$. This type of behavior has also been observed in the ferromagnetic compound $\mathrm{CeAgSb}_{2}$, where the CEF predicts moments in the ab-plane, but the experimental moment direction is along the $c$ axis. ${ }^{45,46}$ As this is a ferromagnet, the change in the easy axis direction can be clearly seen in the temperature dependence of the single-crystal susceptibility, which shows a crossover below the magnetic ordering temperature. ${ }^{46}$ This is not the case for antiferromagnetically ordered systems, where no such crossover is seen. Instead, the easy magnetization axis direction can be determined by examining the temperaturedependent susceptibility, which decreases below $\mathrm{T}_{\mathrm{N}}$ for the direction parallel to the sublattice magnetization, ${ }^{47}$ while the perpendicular susceptibility remains almost constant (or is weakly temperature dependent) below $\mathrm{T}_{\mathrm{N}}$. This is the case for $\mathrm{CeRhGe}_{3}$ and very similar behavior has been observed in $\mathrm{CeRhIn}_{5}$ and $\mathrm{CePt}_{3} \mathrm{Si}^{48-51}$ The neutron diffraction study of $\mathrm{CeRhIn}_{5}$ reveals that the orientation of the magnetic moments is in the ab-plane, while the susceptibility shows an easy 


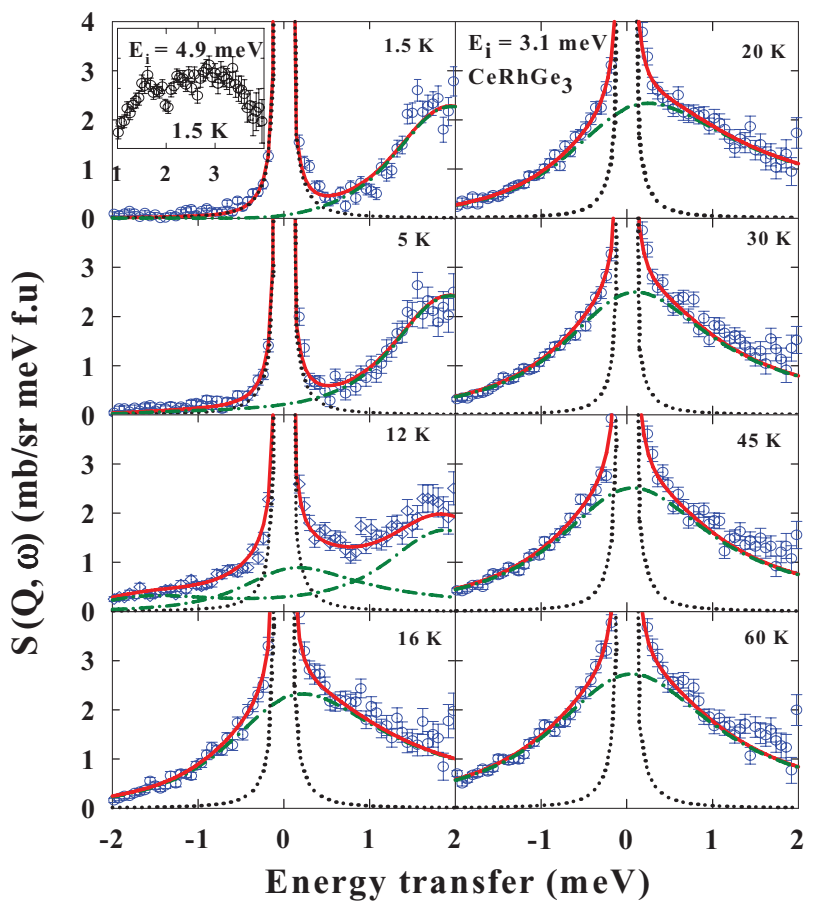

FIG. 11. (Color online) The observed scattering between $10^{\circ}$ and $115^{\circ}$ scattering angles for $\mathrm{CeRhGe}_{3}$ at various temperatures measured with $\mathrm{E}_{i}=3.1 \mathrm{meV}$. The data were fitted using a Lorentzian line-shape function for the quasielastic and inelastic components. The solid line shows the fit and dashed (elastic peak) and dashed-dotted (quasielastic and inelastic peaks) lines represent the components of the fit. The inset (top left) shows the data measured with $\mathrm{E}_{i}=4.9 \mathrm{meV}$ at $1.5 \mathrm{~K}$.

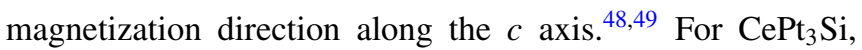
the single-crystal susceptibility and CEF parameters obtained from the INS analysis predict an easy magnetization along the $c$ axis, ${ }^{50}$ but the neutron diffraction data show that the moments are in the ab-plane. ${ }^{51}$ Recently, similar behavior has been observed in $\mathrm{CeRu}_{2} \mathrm{Al}_{10} .{ }^{52}$ These observations suggest that a systematic study, both theoretical and experimental, on Ce-based systems, is highly necessary to obtain a complete understanding of the role of anisotropic exchange and singleion crystal-field anisotropies on the magnetism of these strongly correlated electron systems.

\section{Low-energy INS study}

To elucidate the nature of the low-energy excitations, Kondo temperature and spin-wave energy scale, we have also performed low-energy INS measurements on $\mathrm{CeRhGe}_{3}$, and the results are shown in Fig. 11. The data are plotted as the total scattering from all the detectors for scattering angles from $10^{\circ}$ to $115^{\circ}$. At $1.5 \mathrm{~K}$ the scattering was mainly dominant below $0.7 \AA^{-1}$ and above $1 \mathrm{meV}$, which confirmed its spin-wave origin. In order to cover the high-nergy part of the spin waves, we also carried out measurements with higher $\mathrm{E}_{i}=4.9 \mathrm{meV}$ at $1.5 \mathrm{~K}$, and the integrated scattering is plotted in the inset of Fig. 11 (top left side). It is clear from the inset that the scattering intensity has a peak near $3 \mathrm{meV}$ with a possibility of another peak near $2 \mathrm{meV}$. This suggests the presence of two (or more) possible spin-wave modes. At $5 \mathrm{~K}$ the spin-wave scattering is almost identical to

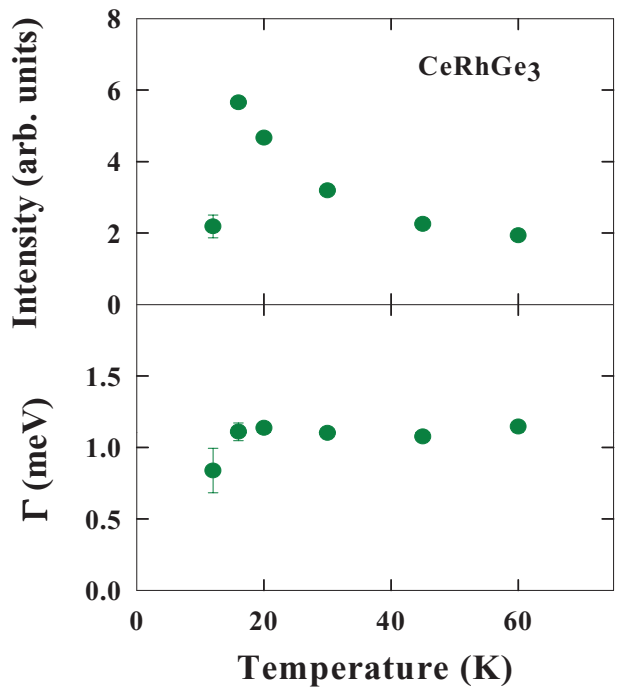

FIG. 12. (Color online) Temperature dependence of the intensity (top) and linewidth (bottom) of the quasielastic line estimated from the fit (see text).

that observed at $1.5 \mathrm{~K}$, but at $12 \mathrm{~K}$, we can see an increase in the quasielastic scattering and a slight decrease in the spin-wave intensity. Considering the numbers of spin-wave modes equal to the numbers of magnetic atoms per magnetic unit cell, one expects two degenerate spin-wave modes for a body-centered tetragonal collinear AFM (type-I AFM) structure (with equal moments on both $\mathrm{Ce}$ atoms, two $\mathrm{Ce}$ atoms per magnetic unit cell) for an isotropic magnetic exchange. It is to be noted that an anisotropic magnetic exchange could lift the degeneracy of the spin-wave modes. Furthermore the spin density wave-type AFM structure of $\mathrm{CeRhGe}_{3}$, having six Ce atoms carrying magnetic moment per magnetic cell (four crystallographic cells), will give three degenerate spin-wave modes for an isotropic magnetic exchange and six modes for an anisotropic magnetic exchange. The observed two (or possibly more) peaks in $\mathrm{CeRhGe}_{3}$ can be attributed to the powder averaging from the ab-plane and along the $c$ axis. However, to find out the real origin of the observed two (or more) peaks in the spin-wave scattering a detailed spin-wave investigation on a single crystal of $\mathrm{CeRhGe}_{3}$ is highly desirable.

In the paramagnetic state, between 16 and $60 \mathrm{~K}$, a clear sign of quasielastic scattering has been observed (Fig. 11). From the data it is evident that the quasielastic linewidth $(\Gamma$, HWHM) does not show any dramatic change with temperature. Further, the Q-dependent intensity in the paramagnetic state follows the square of the $\mathrm{Ce}^{3+}$ form factor. To estimate the temperature dependence of $\Gamma$, we have analyzed the data using a Lorentzian line-shape function convoluted with the elastic line resolution function. The elastic line resolution was modeled with Gaussian and Lorentzian functions and their parameters were estimated (and kept fixed in the analysis of $\Gamma$ ) from the vanadium run under the same conditions. The estimated intensity and linewidth of the quasielastic scattering are plotted in Fig. 12. This shows that $\Gamma(\mathrm{T})$ is nearly temperature independent between 16 and $60 \mathrm{~K}$ and decreases slightly at $12 \mathrm{~K}$. On the other hand, the intensity increases with decreasing temperature and drops at $12 \mathrm{~K}$ (Fig. 12). As at 5 and 
$1.5 \mathrm{~K}$, the quasielastic scattering was absent, and the data were fitted with an inelastic peak with fixed position at $1.8 \mathrm{meV}$. By extrapolating $\Gamma(\mathrm{T})$ to $\mathrm{T}=0 \mathrm{~K}$, which gives the measure of $\mathrm{T}_{\mathrm{K}}$, we have estimated $\mathrm{T}_{\mathrm{K}}=12.6(3) \mathrm{K}$ for $\mathrm{CeRhGe}_{3}$, which is in excellent agreement with that estimated using the heat capacity data in Sec. III C.

\section{CONCLUSIONS}

We have used magnetic susceptibility, heat capacity, neutron powder diffraction, INS, and muon spin relaxation studies to probe the magnetic ground state of $\mathrm{CeRhGe}_{3}$. We have observed two magnetic transitions in the heat capacity and susceptibility near 7 and $14.5 \mathrm{~K}$. Our neutron diffraction measurements at $1.5 \mathrm{~K}$ reveal a spin-density-wave-type magnetic ordering with propagation vector $\mathbf{k}=\left(\begin{array}{lll}0 & 0 & \frac{3}{4}\end{array}\right)$ and a moment of $0.45(9) \mu_{\mathrm{B}}$ along the $c$ axis. The muon spin relaxation ( $\mu \mathrm{SR})$ measurements reveal the presence of internal fields below $14.5 \mathrm{~K}$, and we have observed three clear frequencies in the $\mu \mathrm{SR}$ spectra in the temperature range of 7 to $14.5 \mathrm{~K}$, which then merge into two frequencies as the temperature is decreased below $7 \mathrm{~K}$. We attribute these three frequencies to a complex magnetic structure. At $1.5 \mathrm{~K}$, we observed only two frequencies, in agreement with the neutron diffraction data. Both studies confirm that the ground state of $\mathrm{CeRhGe}_{3}$ exhibits long-range magnetic order. From the temperature dependence of the $\mu$ SR frequencies, we have estimated the value of the critical exponent $\beta \sim 0.5$. Further, our high-energy INS study at $18 \mathrm{~K}$ reveals the presence of two well-defined
CEF excitations. The observed direction of the moment along the $c$ axis does not agree with that predicted by the single-ion CEF anisotropy estimated from the analysis of the INS data. This indicates that the direction of the moment is governed by some other anisotropies, probably two-ion anisotropic magnetic exchange as evident from the anisotropic molecular field parameters. A similar behavior has been observed in many other Ce-based HF systems and calls for more detailed experimental and theoretical investigations in these compounds. Our low-energy INS study reveals spin waves below $T_{N}$ and well-defined quasielastic scattering above $T_{N}$. Finally, the comparison of the linewidth of the CEF excitations of $\mathrm{CeRhGe}_{3}$ and $\mathrm{CeRhSi}_{3}$ indicates a stronger hybridization in the latter case, which becomes a superconductor at $2 \mathrm{GPa}$, while no $\mathrm{SC}$ has been observed in $\mathrm{CeRhGe}_{3}$ up to $8 \mathrm{GPa}$. This suggests that the role of the hybridization could be critical for the $\mathrm{SC}$ in $\mathrm{CeTX}_{3}$ compounds. This is further supported through an onset of $\mathrm{SC}$ at ambient pressure in $\mathrm{CeCoSi}_{3}$, which has strongest hybridization among $\mathrm{CeTX}_{3}$ systems.

\section{ACKNOWLEDGMENTS}

We should like to thank Y. Ōnuki, T. Kawai, and F. Honda for providing the single-crystal susceptibility data of $\mathrm{CeRhGe}_{3}$. We also thank R. Smith and W. Kockelmann for carrying out the neutron diffraction measurements on the GEM diffractometer at $300 \mathrm{~K}$, and E. A. Goremychkin, D. Khalyavin, M. Rotter, L. C. Chapon, and B. Lake for interesting discussions. We are grateful to the CMPC-STFC for providing research funding (Grant No. CMPC-09108). *adrian.hillier@stfc.ac.uk

$\dagger$ devashibhai.adroja@stfc.ac.uk

${ }^{1}$ Q. Si, in Understanding Quantum Phase Transitions, edited by Lincoln D. Carr (Taylor \& Francis, Boca Raton, 2011).

${ }^{2}$ T. Park and J. D. Thompson, New J. Phys. 11, 055062 (2009).

${ }^{3}$ C. Pfleiderer, Rev. Mod. Phys. 81, 1551 (2009).

${ }^{4}$ M. Imada and T. Miyake, J. Phys. Soc. Jpn. 79, 112001 (2010).

${ }^{5}$ N. D. Mathur, F. M. Grosche, S. R. Julian, I. R. Walker, D. M. Freye, R. K. W. Haselwimmer, and G. G. Lonzarich, Nature (London) 394, 39 (1998).

${ }^{6}$ V. K. Anand, A. D. Hillier, D. T. Adroja, A. M. Strydom, H. Michor, K. A. McEwen, and B. D. Rainford, Phys. Rev. B 83, 064522 (2011).

${ }^{7}$ D. T. Adroja, A. D. Hillier, V. K. Anand, E. A. Goremychkin,

K. A. McEwen, B. D. Rainford, V. V. Krishnamurthy, and G. Balakrishnan, ISIS Facility Experimental Report, RB820238, 2009 (unpublished)

${ }^{8}$ V. K. Anand, D. T. Adroja, A. D. Hillier, and W. A. Kockelmann, J. Phys. Condens. Matter 23, 276001 (2011)

${ }^{9}$ V. K. Anand, D. T. Adroja, A. D. Hillier, J. Taylor, and G. André, Phys. Rev. B 84, 064440 (2011).

${ }^{10}$ P. Haen, P. Lejay, B. Chevalier, B. Lloret, J. Etourneau, and M. Sera, J. Less-Common Met. 110, 321 (1985).

${ }^{11}$ Y. Iwamoto, K. Ueda, T. Kohara, and Y. Yamada, Physica B 206 \& 207, 276 (1995).

${ }^{12}$ N. Kimura, K. Ito, K. Saitoh, Y. Umeda, H. Aoki, and T. Terashima, Phys. Rev. Lett. 95, 247004 (2005).
${ }^{13}$ Y. Muro, M. Ishikawa, K. Hirota, Z. Hiroi, N. Takeda, N. Kimura, and H. Aoki, J. Phys. Soc. Jpn. 76, 033706 (2007).

${ }^{14}$ I. Sugitani, Y. Okida, H. Shishido, T. Yamada, A. Thamizhavel, E. Yamamoto, T. D. Matsuda, Y. Haga, T. Takeuschi, R. Settai, and Y. Ōnuki, J. Phys. Soc. Jpn. 75, 043703 (2006).

${ }^{15}$ H. Mukuda, T. Fujii, T. Ohara, A. Harada, M. Yashima, Y. Kitaoka, Y. Okuda, R. Settai, and Y. Ōnuki, Phys. Rev. Lett. 100, 107003 (2008).

${ }^{16}$ Y. Muro, D. Eom, N. Takeda, and M. Ishikawa, J. Phys. Soc. Jpn. 67, 3601 (1998).

${ }^{17}$ T. Kawai, H. Murankai, M. A. Meassoni, T. Shimodai, Y. Doii, T. D. Matsuda, Y. Haga, G. Knebel, G. Lapertot, D. Aoki, J. Flouquet, T. Takeuchi, R. Settai, and Y. Ōnuki, J. Phys. Soc. Jpn. 77, 064716 (2008).

${ }^{18}$ F. Honda, I. Bonalde, K. Shimizu, S. Yoshiuchi, Y. Hirose, T. Nakamura, R. Settai, and Y. Ōnuki, Phys. Rev. B 81, 140507R (2010).

${ }^{19}$ T. Kawai, Y. Okuda, H. Shishido, A. Thamizhavel, T. D. Matsuda, Y. Haga, M. Nakashima,T. Takeuchi, M. Hedo, Y. Uwatoko, R. Settai, and Y. Ônuki, J. Phys. Soc. Jpn. 76, 014710 (2007).

${ }^{20}$ A. Thamizhavel, T. Takeuchi, T. D. Matsuda, Y. Haga, K. Sugiyama, R. Settai, and Y. Ōnuki, J. Phys. Soc. Jpn. 74, 1858 (2005).

${ }^{21}$ R. Settai, I. Sugitani, Y. Okuda, A. Thamizhavel, M. Nakashima, Y. Ōnuki, and H. Harima, J. Magn. Magn. Mater. 310, 844 (2007); K. Kaneko, N. Metoki, T. Takeuchi, T. D. Matsuda, Y. Haga, A. Thamizhavel, R. Settai, and Y. Ōnuki, J. Phys. Conf. Ser. 150, 042082 (2009). 
${ }^{22}$ M. Nakashima, K. Tabata, A. Thamizhavel, T. C. Kobayashi, M. Hedo, Y. Uwatoko, K. Shimizu, R. Settai, and Y. Ōnuki, J. Phys. Condens. Matter 16, L255 (2004).

${ }^{23}$ H. Kotegawa, K. Takeda, T. Miyoshi, S. Fukushima, H. Hidaka, T. C. Kobayashi, T. Akazawa, Y. Ohishi, M. Nakashima, A. Thamizhavel, R. Settai, and Y. Ōnuki, J. Phys. Soc. Jpn. 75, 044713 (2006);

A. Harada, H. Mukuda, Y. Kitaoka, A. Thamizhavel, Y. Okuda, R. Settai, Y. Ōnuki, K. M. Itoh, E. E. Haller, and H. Harima, Physica B 403, 1020 (2008).

${ }^{24}$ L. C. Chapon, P. Manuel, P. Radaelli, C. Benson, L. Perrott, S. Ansell, N. J. Rhodes, D. Raspino, D. Duxbury, E. Spill, and N. Norris, Neutron News 22, 22 (2011).

${ }^{25}$ V. K. Anand, Z. Hossain, and C. Geibel, Solid State Commun. 146, 335 (2008).

${ }^{26}$ G. Grüner and A. Zawadowski, Rep. Prog. Phys. 37, 1497 (1974).

${ }^{27}$ H. R. Krishna-Murthy, J. W. Wilkins, and K. G. Wilson, Phys. Rev. B 21, 1003 (1980).

${ }^{28}$ E. Bauer, N. Pillmayr, E. Gratz, and G. Hilscher, Z. Phys. B 67, 205 (1987).

${ }^{29}$ P. K. Das, N. Kumar, R. Kulkarni, and A. Thamizhavel, Phys. Rev. B 83, 134416 (2011).

${ }^{30}$ B. M. Sondezi-Mhlungu, D. T. Adroja, A. M. Strydom, S. Paschen, and E. A. Goremychkin, Physica B 404, 3032 (2009).

${ }^{31}$ D. T. Adroja, A. D. Hillier, and G. André, (unpublished).

${ }^{32}$ M. J. Besnus, A. Braghta, N. Hamdaoui, and A. Meyer, J. Magn. Magn. Mater. 104-107, 1385 (1992).

${ }^{33}$ J. A. Blanco, M. de Podesta, J. I. Espeso, J. C. Gómez Sal, C. Lester, K. A. McEwen, N. Patrikios, and J. Rodríguez Fernández, Phys. Rev. B 49, 15126 (1994).

${ }^{34}$ S. V. Vonsovskii, Magnetism (Wiley, New York, 1974).

${ }^{35}$ The detailed information can be obtained from Inorganic Crystal Structure Database (ICSD), [http//cds.dl.ac.uk/], D. A. Fletcher, R. F. McMeeking, and D. Parkin, J. Chem. Inf. Comput. Sci. 36, 746 (1996).

${ }^{36}$ H. T. Stokes, D. M. Hatch, and B. J. Campbell, ISOTROPY, software package, [http://stokes.byu.edu/iso/isotropy.html] (2007).

${ }^{37}$ O. Kovalev, Representation of the Crystallographic Space Groups (Gordon and Breach Science Publishers, London, 1993).

${ }^{38}$ J. Rodriguez-Carvajal, Physica B 192, 55 (1993).

${ }^{39}$ P. Thalmeier, J. Phys. C 17, 4153 (1984).

${ }^{40}$ D. T. Adroja, A. del Moral, C. de la Fuente, A. Fraile, E. A. Goremyhkin, J. W. Taylor, A. D. Hillier and F. Fernandez-Alonso, submitted to Phys. Rev. Lett. (2012).

${ }^{41}$ K. W. H. Stevens, Proc. Phys. Soc. London, Sect. A 65, 209 (1952); Rep. Prog. Phys. 30, 189 (1976); Phys. Rep. 24, 1 (1976).

${ }^{42}$ J. Jensen and A. R. Mackintosh, Rare Earth Magnetism (Clarendon Press, Oxford, 1991).

${ }^{43}$ G. Marusi, N. V. Mushnikov, L. Paretit, M. Solzi, and A. E. Ermakov, J. Phys. Cond. Matt. 2, 7317 (1990).

${ }^{44}$ W. Zheng, R. R. P. Singh, R. H. McKenzie, and R. Coldea, Phys. Rev. B 71, 134422 (2005); M. Doerr, A. Haase, M. Loewenhaupt,
M. Rotter, M. Bartkowiak, R. Daou, E. Kampert, J. A. A. J. Perenboom, and T. Tsutaoka, ibid. 82, 024422 (2010).

${ }^{45}$ G. André, F. Bourée, M. Kolenda, B. Leśniewska, A. Oleś, and A. Szytula, Physica B 292, 176 (2000); T. Takeuchi, A. Thamizhavel, T. Okubo, M. Yamada, N. Nakamura, T. Yamamoto, Y. Inada, K. Sugiyama, A. Galatanu, E. Yamamoto, K. Kindo, T. Ebihara, and Y. Ōnuki, Phys. Rev. B 67, 064403 (2003).

${ }^{46}$ K. D. Myers, S. L. Bud'ko, I. R. Fisher, Z. Islam, H. Kleinke, A. H. Lacerda, and P. C. Canfield, J. Magn. Magn. Mater. 205, 27 (1999).

${ }^{47}$ N. W. Ashcroft and N. D. Mermin, Solid State Physics (W. B. Saunders Company, Philadelphia, 1976), p. 702.

${ }^{48}$ H. Hegger, C. Petrovic, E. G. Moshopoulou, M. F. Hundley, J. L. Sarrao, Z. Fisk, and J. D. Thompson, Phys. Rev. Lett. 84, 4986 (2000).

${ }^{49}$ W. Bao, P. G. Pagliuso, J. L. Sarrao, J. D. Thompson, Z. Fisk, J. W. Lynn, and R. W. Erwin, Phys. Rev. B 62, R14621 (2000);

T. Willers, Z. Hu, N. Hollmann, P. O. Körner, J. Gegner, T. Burnus, H. Fujiwara, A. Tanaka, D. Schmitz, H. H. Hsieh, H.-J. Lin, C. T. Chen, E. D. Bauer, J. L. Sarrao, E. Goremychkin, M. Koza, L. H. Tjeng, and A. Severing, ibid. 81, 195114 (2010).

${ }^{50}$ N. Metoki, K. Kaneko, T. D. Matsuda, A. Galatanu, T. Takeuchi, S. Hashimoto, T. Ueda, R. Settai, Y. Onuki, and N. Bernhoeft, J. Phys. Condens. Matter 16, L207 (2004).

${ }^{51}$ E. Bauer, G. Hilscher, H. Michor, M. Sieberer, E. W. Scheidt, A. Gribanov, Yu. Seropegin, P. Rogl, A. Amato, W. Y. Song, J.-G. Park, D. T. Adroja, M. Nicklas, G. Sparn, M. Yogi, and Y. Kitaoka, Physica B 359-361, 360 (2005).

${ }^{52}$ D. D. Khalyavin, A. D. Hillier, D. T. Adroja, A. M. Strydom, P. Manuel, L. C. Chapon, P. Peratheepan, K. Knight, P. Deen, C. Ritter, Y. Muro, and T. Takabatake, Phys. Rev. B 82, 100405 (2010); H. Tanida, D. Tanaka, M. Sera, C. Moriroshi, Y. Kuroiwa, T. Takesaka, T. Nishioka, H. Kato, and M. Matsumura, J. Phys. Soc. Jpn. 79, 083701 (2010); J. M. Mignot, J. Robert, G. André, A. M. Bataille, T. Nishioka, R. Kobayashi, M. Matsumura, H. Tanida, D. Tanaka, and M. Sera, ibid. 80, SA022 (2011).

${ }^{53}$ P. J. Brown, in International Tables for Crystallography, edited by A. J. C. Wilson, (Kluwer Academic, Amsterdam 1999), Vol. C, pp. $450-457$.

${ }^{54}$ D. Eom, M. Ishikawa, J. Kitagawa, and N. Takeda, J. Phys. Soc. Jpn. 67, 2495 (1998).

${ }^{55}$ J. Kitagawa, Y. Muro, N. Takeda, and M. Ishikawa, J. Phys. Soc. Jpn. 66, 2163 (1997).

${ }^{56}$ H. Yamamoto, M. Ishikawa, K. Hasegawa, and J. Sakurai, Phys. Rev. B 52, 10136 (1995).

${ }^{57}$ A. P. Pikul, D. Kaczorowski, T. Plackowski, A. Czopnik, H. Michor, E. Bauer, G. Hilscher, P. Rogl, and Yu. Grin, Phys. Rev. B 67, 224417 (2003).

${ }^{58}$ K. Ghosh, S. Ramakrishnan, S. K. Dhar, S. K. Malik, G. Chandra, V. K. Pecharsky, K. A. Gschneidner Jr., Z. Hu, and W. B. Yelon, Phys. Rev. B 52, 7267 (1995). 